\title{
Pile-Reinforcement Behavior of Cohesive Soil Slopes: Numerical Modeling and Centrifuge Testing
}

\author{
Liping Wang and Ga Zhang \\ State Key Laboratory of Hydroscience and Engineering, Tsinghua University, Beijing 100084, China \\ Correspondence should be addressed to Ga Zhang; zhangga@tsinghua.edu.cn
}

Received 6 June 2013; Accepted 29 July 2013

Academic Editor: Fayun Liang

Copyright ( 2013 L. Wang and G. Zhang. This is an open access article distributed under the Creative Commons Attribution License, which permits unrestricted use, distribution, and reproduction in any medium, provided the original work is properly cited.

Centrifuge model tests were conducted on pile-reinforced and unreinforced cohesive soil slopes to investigate the fundamental behavior and reinforcement mechanism. A finite element analysis model was established and confirmed to be effective in capturing the primary behavior of pile-reinforced slopes by comparing its predictions with experimental results. Thus, a comprehensive understanding of the stress-deformation response was obtained by combining the numerical and physical simulations. The response of pile-reinforced slope was indicated to be significantly affected by pile spacing, pile location, restriction style of pile end, and inclination of slope. The piles have a significant effect on the behavior of reinforced slope, and the influencing area was described using a continuous surface, denoted as $W$-surface. The reinforcement mechanism was described using two basic concepts, compression effect and shear effect, respectively, referring to the piles increasing the compression strain and decreasing the shear strain of the slope in comparison with the unreinforced slope. The pile-soil interaction induces significant compression effect in the inner zone near the piles; this effect is transferred to the upper part of the slope, with the shear effect becoming prominent to prevent possible sliding of unreinforced slope.

\section{Introduction}

Landslides are one of the severest geologic hazards around the world, the prevention of which is of great interest to engineers and researchers. The stabilizing pile, an important reinforcement structure, has been widely used to support unstable slopes in the last few decades [1-3]. Many methods have been proposed to form a good basis for proper design of pile-reinforced slopes $[4,5]$; however, there are a few important issues to be clarified for the application of such piles in slope engineering. Therefore, systematical investigations are required on the behavior and reinforcement mechanism of pile-reinforced slopes.

The behavior of pile-reinforced slope can be investigated by using a diverse range of research approaches, which can be generally divided into three categories: field observations, model tests, and numerical analyses. Field observation is an essential approach to obtain first-hand data of reinforced slopes. For example, long-term monitoring was used to analyze the bending moments and displacements of the piles that were employed for a railway embankment [2]. Nevertheless, boundary conditions or loading conditions cannot be easily changed in a field test, which restricts such an approach to the study of the reinforcement mechanism.

Model tests offer a powerful approach to investigate the behavior and failure mechanism of a reinforced slope by efficiently considering various factors. A series of $1 \mathrm{~g}$ model tests were used to investigate the behavior of a pile-stabilized sandy slope $[6,7]$. The centrifuge model tests play an important role in such a category because they provide an accurate simulation of the gravity stress field and the gravity-related deformation process. Therefore, centrifuge modeling has been widely used to study reinforced slopes with different reinforcement structures, including piles, geomembranes, geotextiles, and soil nails [8-13]. The measurement was finite in the model tests due to the small size of the model and the limitation of measurement technology; for example, the stress state of the slope cannot be measured with sufficient accuracy.

Numerical analysis can yield comprehensive information about the response of the slope; thus, a few different types 
of numerical methods were developed or used to study the reinforced slopes, such as the limit equilibrium method $[14,15]$, limit analysis [16], the finite-element method [17], and other rigorous or simplified methods [18]. For example, Won et al. compared the predictions by limit equilibrium analysis and three-dimensional numerical analysis involving a shear strength reduction technique for a slope-pile system [19]. A three-dimensional numerical analysis was used to investigate the influence of sleeving on the pile performance in a sloping ground $[20,21]$. The FLAC3D program was used to analyze the response of piles in an embankment slope with a translational failure mode, and the results showed that the pile-soil relative stiffness has a significant influence upon the piles' failure mode [22]. Except for the algorithm, the effectiveness of numerical analysis of reinforced slope is also significantly affected by several aspects, such as the soil model, the soil-structure interface model, and their parameters, which should be acknowledged not to be sufficiently reliable due to the complexity of this problem.

Most previous investigations of the pile-reinforced slope focused on the response of the slope and piles as well as on the influence factors; from those, a few useful conclusions have been achieved. However, the response of the pile-reinforced slope under various types of load applications has not been illustrated in a fully comprehensive view. Moreover, the stabilizing mechanism-for example, why the avoidance of failure is induced by the piles-has not yet been adequately discovered. In other words, further study is needed to clarify how the local pile-soil interaction affects the deformation field of the entire slope and, therefore, increases the stability level. In addition, the deformation trends and main influence factors of the behavior of pile-reinforced slopes have not been fully discovered.

Based on the understanding of the main features of the numerical methods and model tests, an effective approach may be to combine both of the above methods to acquire a comprehensive description of the pile-reinforced slopes. Therefore, the observations from the model tests can be supplemented by a numerical method that has been verified by the model tests. The objective of this paper is to conduct such an attempt for the purpose of reinforcement mechanism analysis of pile-reinforced slope, including (1) to conduct centrifuge model tests of a cohesive soil slope using stabilizing piles, in comparison with the unreinforced slope; (2) to present a numerical scheme of the pile-reinforced slope and to confirm its effectiveness by simulating the centrifuge tests; (3) to analyze the behavior of the reinforced slope by using the test observations and numerical analysis; (4) to describe the reinforcement mechanism; and (5) to discuss the main factors that influence the behavior of reinforced slopes.

\section{Centrifuge Model Tests}

The centrifuge model tests were conducted using the $50 \mathrm{~g}$ ton geotechnical centrifuge at Tsinghua University.

2.1. Model. The soil of the slope model was retrieved directly from the soil mountain of the Beijing Olympic Forest Park.
The average grain size of the soil is $0.03 \mathrm{~mm}$, and the plastic limit and liquid limit are 5\% and 18\%, respectively.

Figure 1 shows the photographic and schematic views of the model slope, reinforced using stabilizing piles. The unreinforced slope is identical except for removal of the piles. The model container for the tests, made of aluminum alloy, is $50 \mathrm{~cm}$ long, $20 \mathrm{~cm}$ wide, and $35 \mathrm{~cm}$ high. A transparent Lucite window was installed in one container side to observe the deformation process of the soil.

The soil was compacted into the container by a $6-\mathrm{cm}-$ thickness layer, with a dry density and water content of $1.4 \mathrm{~g} / \mathrm{cm}^{3}$ and $16 \%$, respectively. The slope was obtained by removing the redundant soil, with an inclination of $1.5: 1$ and height of $25 \mathrm{~cm}$. A $6 \mathrm{~cm}$ high horizontal soil layer under the slope was set to diminish the influence of the bottom container. In addition, silicone oil was painted on both sides of the container to decrease the friction on the slope.

A hollow square pipe, made of steel with an elastic modulus of $210 \mathrm{GPa}$, was used to simulate the stabilizing pile of the reinforced slope. The pipe was $1.4 \mathrm{~cm}$ along the side length of the section, with a wall thickness of $1.5 \mathrm{~mm}$. This is equivalent to a prototype pile with a side length of $0.7 \mathrm{~m}$ at $50 \mathrm{~g}$-level. These piles were inserted in the slope, without special fixation, at a single row $10 \mathrm{~cm}$ apart and $6 \mathrm{~cm}$ far away from the slope toe. Half-section piles were used near both container sides to approximate the plane-strain condition (Figure 1(c)).

2.2. Measurements. An image-record and displacement measurement system was used to record the images of soil during the centrifuge tests [23], which are used to determine the displacement history of an arbitrary point of soil, without disturbing the soil itself, by an image-correlation-analysis algorithm [24]. The effectiveness of this measurement system was realized by embedding white particles laterally in the soil (Figure 1(a)). The measurement accuracy can reach $0.02 \mathrm{~mm}$ based on the model dimension for the centrifuge tests in this paper. In addition, a few patterns with significant grey difference were affixed onto the pile to obtain displacement history of the pile on the basis of image analysis. The area within the dotted line was used for displacement measurements owing to the requirement of the measurement system (Figure 1(b)); the main deformation zone can be covered. Cartesian coordinates were established with the origin as the intersection between the slope bottom and inner sidewall of the pile; positive directions were specified (Figures 1(b) and 1(c)).

Four pairs of strain gauges were attached to the inner walls of the middle pile to measure the strain distribution along the shaft (Figure 1(b)). They can be used to derive the bending moment and axial load of the pile.

2.3. Test Procedure. The model slope was installed on the centrifuge machine, and increasing centrifugal acceleration was applied at steps of $5 \mathrm{~g}$. Each acceleration step was maintained for several minutes until the deformation of the slope became stable. This process was terminated at $50 \mathrm{~g}$-level when the unreinforced slope exhibited significant failure.

2.4. Observations. It should be noted that all of the results are based on the model dimension in this paper. Figure 2 


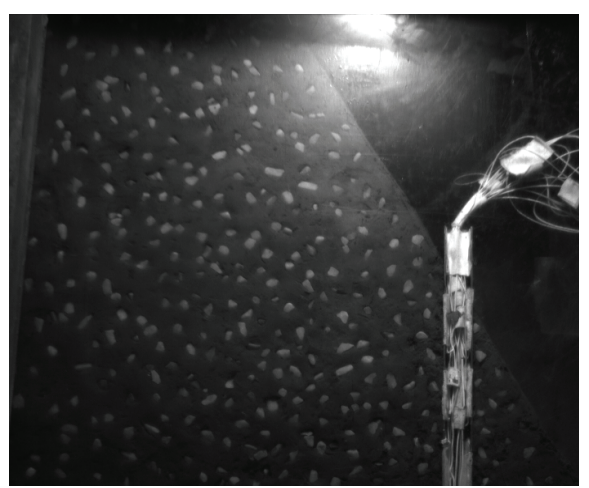

(a) Photograph

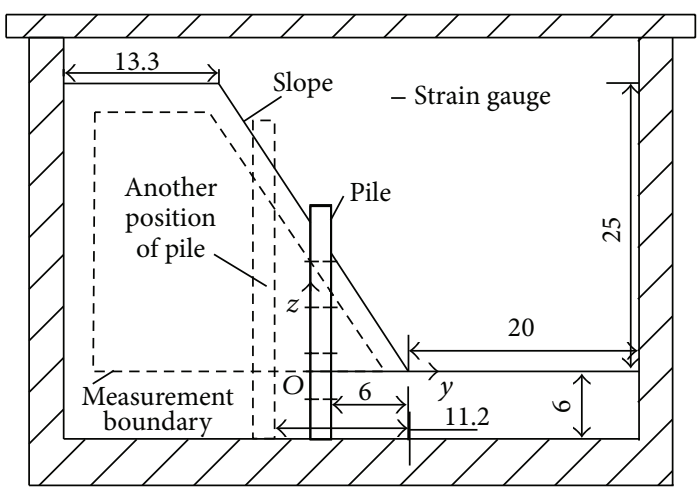

(b) Elevation view

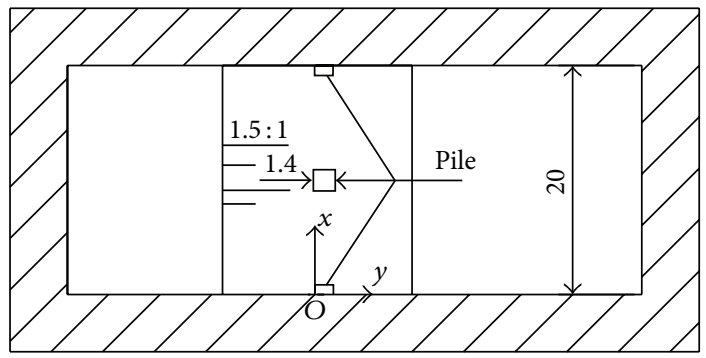

(c) Vertical view

FIGURE 1: Schematic view of the model of reinforced slope (unit: $\mathrm{cm}$ ).

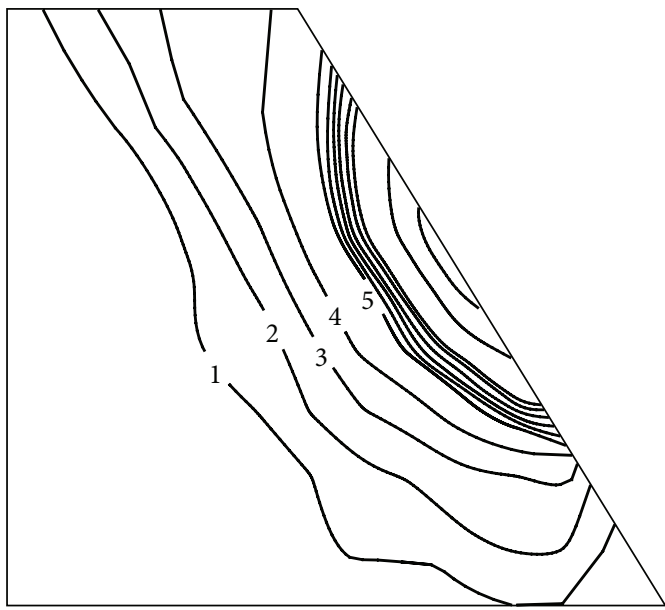

(a) Unreinforced slope at failure state

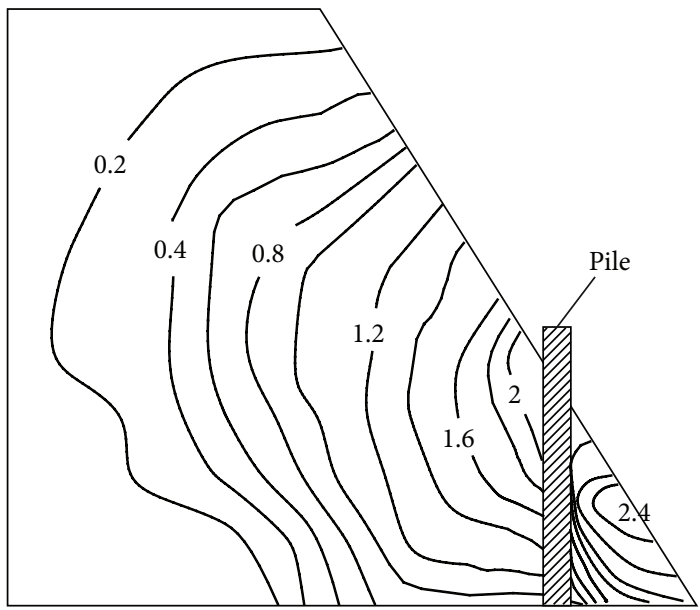

(b) Pile-reinforced slope

FIGURE 2: Horizontal displacement contours of slopes at 50 g-level (unit: mm).

shows the horizontal displacement distribution of the pile-reinforced and unreinforced slopes at $50 \mathrm{~g}$-level; the borders in the figures were designated as the dotted area in Figure 1(b), but these do not correspond to the actual slope borders. It can be seen that a significant landslide occurred in the unreinforced slope when the centrifuge acceleration reached $50 \mathrm{~g}$ (Figure 2(a)): there was significant concentration of deformation so that the slide body can be easily distinguished from the base body via the contour lines. On the other hand, the reinforced slope only exhibited significant deformation due to the increase of centrifugal acceleration; a landslide was avoided at $50 \mathrm{~g}$-level (Figure 2(b)). This indicated that the piles significantly increased the stability level of the slope. Moreover, the deformation of the reinforced slope was significantly smaller than that of the unreinforced slope, demonstrating that the piles had a significant effect on the deformation of the slope.

\section{Numerical Analysis}

3.1. Analysis Model. A three-dimensional finite element method was used to simulate the pile-reinforced slope under 
the condition of the centrifuge model test based on geotechnical stress-strain FEM software, termed TOSS3D, that has been widely used for embankments in China [25]. A new iterative routine was developed to simulate the successive increase of centrifugal acceleration in the software. The explicit increment scheme was used in the nonlinear static $\mathrm{FE}$ analysis. A substep was divided into several subincrements to simulate the nonlinear loading. An iterative algorithm was employed to obtain the stress-strain states of the slope within a subincrement, with a trial algorithm used to judge loading states of the geomaterials and contact states of the interface.

A three-dimensional FE mesh was established with accurate simulation of the slope model for centrifuge tests (Figure 3). The soil was described using hexahedron elements with eight nodes. The soil was described using an elastoplasticity model that can reasonably capture the dilatancy behavior of the soil [25]. The model parameters were determined from triaxial compression tests and adjusted slightly in the numerical analysis for a better fit to the test observations. The parameters and their values are listed in Table 1 and their definitions could be referred to [25]. It should be noted that the cohesion strength parameter, $c$, was a bit greater than the empirical. This may be partially because the boundary effect on the model slope in the centrifuge model tests was considered by the strength parameters of the soil. The interface elements were set between the pile and neighboring soil and between the container sides and neighboring soil. The interface was described using an elastoplasticity damage model, which provides a unified description of monotonic and cyclic behavior, including volumetric behavior [26]. This model was used for many soil-structure systems, such as high concretefaced rockfill dams [27]. The model parameters were determined by a series of shear tests under constant normal stress conditions. The parameters and their values are listed in Table 2 and their definitions could be referred to [26]. The pile was described using a linear elastic model, with elastic modulus of $210 \mathrm{GPa}$ and Poisson's ratio of 0.3 . A soft element set on the pile end to realize the movement of pile. Moreover, another case, with the pile located in the upper slope, as shown in Figure 1(b), was also considered in the mesh. The boundaries of the model slope were all fixed (Figure 3). The mesh was finally obtained according to the symmetry of this problem, involving 8448 nodes and 7140 elements in total.

3.2. Verification. The numerical predictions of displacement response of the reinforced slope were compared with the measurements of the centrifuge model test to verify the effectiveness of the numerical analysis.

Figure 4 shows a comparison of test results and numerical predictions of the contours of displacement of the reinforced slope at $50 \mathrm{~g}$-level. It can be seen that the predicted curve showed a good fit to the test result; this demonstrates that the numerical method provides a reasonable description of the overall performance of the slope. Close comparisons of displacement distribution between the test results and numerical predictions were made on several vertical sections (Figure 5). The horizontal and vertical displacements exhibited the maximum at the middle and top of the slope, respectively. These comparisons showed that the numerical prediction
TABLE 1: Model parameters of the elastoplasticity model for soil.

\begin{tabular}{lccccccc}
\hline$c(\mathrm{kPa})$ & $\phi_{0}\left({ }^{\circ}\right)$ & $R_{f}$ & $K$ & $n$ & $G$ & $F$ & $D$ \\
\hline 50 & 35 & 0.7 & 92 & 0.15 & 0.05 & 0.15 & 1.5 \\
\hline
\end{tabular}

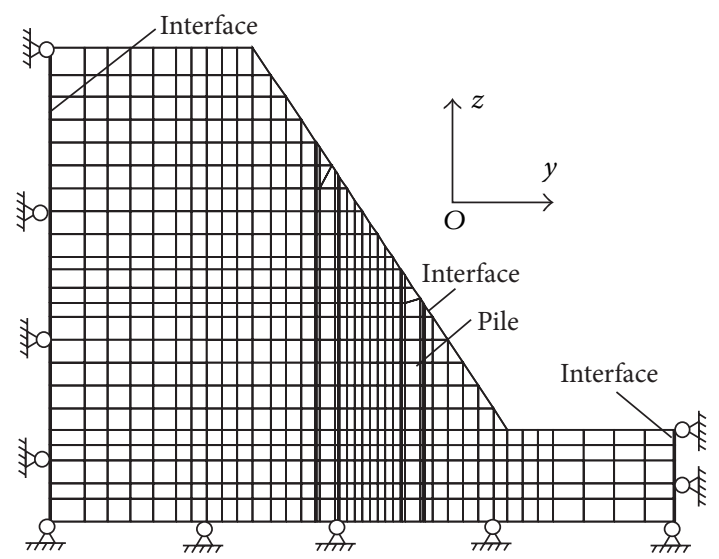

(a) Elevation view

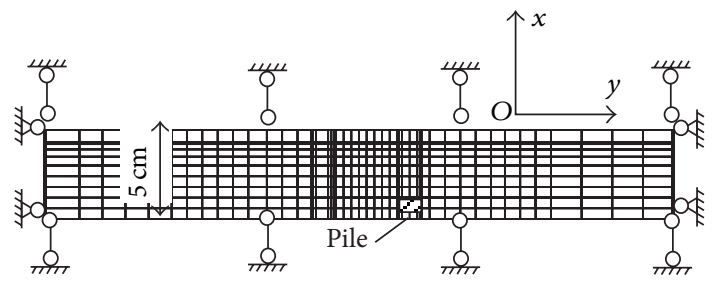

(b) Vertical view

Figure 3: Mesh and boundary of the slope for numerical analysis.

curves were in satisfactory agreement with the test results at different locations. In addition, the vertical displacement histories of a typical point of the slope indicated that the vertical displacement increased with increasing centrifugal acceleration (Figure 6), and the numerical prediction showed a good fit to the test observation.

The response of the pile was also used for the comparison of numerical analysis and test results (Figure 7); a satisfactory fit can be found. It should be noted that we used the difference between the vertical strains obtained from the strain gauges on the left and right sides of the pile to consider the bending behavior (Figure 7(b)); this difference exhibited the maximum at the middle part.

According to the comparison results, it can be concluded that the numerical analysis is effective in capturing the primary behavior of a pile-reinforced slope. The numerical results can be further used to analyze the stress response of the reinforced slope, which is important for understanding the reinforcement behavior but difficult to be measured in the centrifuge model tests. Thus, a comprehensive stressdeformation response can be obtained by combining the numerical and physical simulations.

\section{Stress-Deformation Behavior}

The numerical results have shown that the stress-displacement response of the reinforced slope is approximated for 
TABLE 2: EPDI model parameters of the interface.

\begin{tabular}{lccccccccccc}
\hline$\varepsilon_{\mathrm{vd}, \mathrm{i}, \mathrm{ult}}$ & $\alpha$ & $\beta$ & $\varphi_{0}\left(^{\circ}\right)$ & $G_{0}$ & $n_{0}$ & $\mu_{0}$ & $m_{0}$ & $k_{0}$ & $m_{k 0}$ & $C_{e}$ & $C_{0}$ \\
\hline 0.15 & 500 & 1.0 & 33 & 30 & 0.5 & 300 & 0.3 & 0 & 0.1 & 0.005 & 0.005 \\
\hline
\end{tabular}
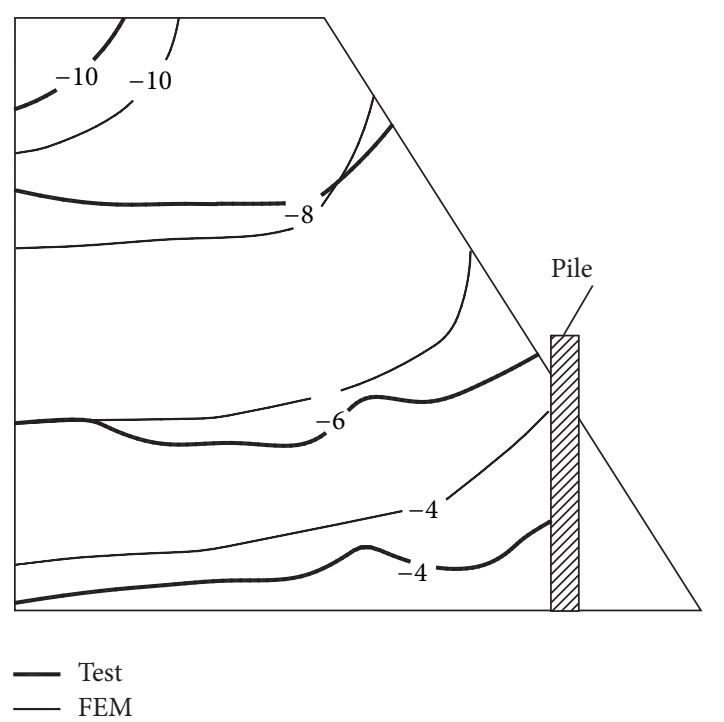
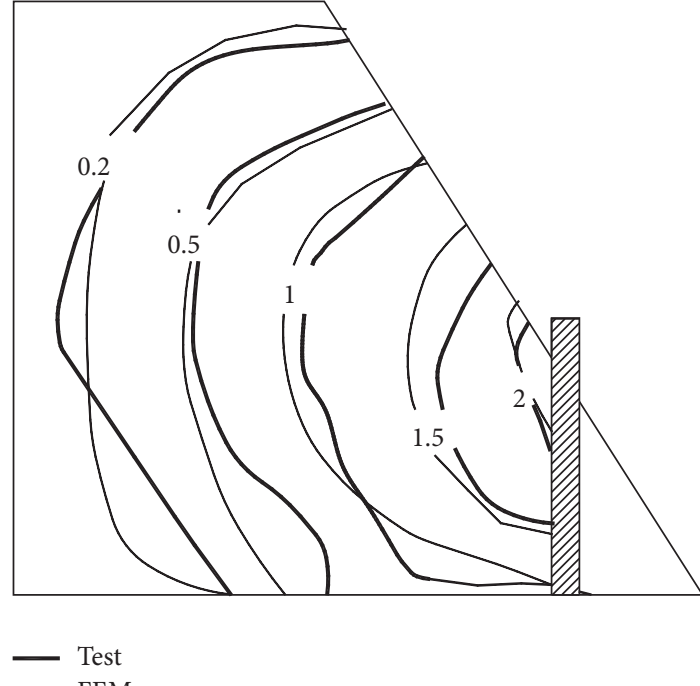

(b) Horizontal displacement

FIGURE 4: Displacement distribution comparison of numerical analysis and test results (unit: $\mathrm{mm}$ ).

different sections, whether the piles pass through or not if the pile space is not large, as for most practical cases. Therefore, the behavior of the pile-reinforced slope was analyzed based mainly on the stress-displacement distribution of the lateral side of the slope that the pile passed through, so that the measurement results of the centrifuge model test can be combined with the predictions of numerical analysis.

The stress distribution of the slope was illustrated using numerical analysis at $50 \mathrm{~g}$-level (Figure 8); there was significant stress concentration near the piles. This demonstrated that the piles had a significant effect on the stress state of the neighboring slope, as did the displacement measured by the tests (Figure 2). Figure 9 shows the stress histories of a typical point on the slope. The magnitude of stress increased with increasing centrifugal acceleration; similar rules can be found in the displacement histories (Figure 6). It can be concluded that the stress-deformation response at $50 \mathrm{~g}$-level can be used as a representative time for further analysis.

4.1. Significant Influence Surface. The piles have a more significant influence on the horizontal displacement of the slope than on the vertical displacement, according to the comparison of reinforced and unreinforced slopes. Thus, the distributions of horizontal displacement at horizontal lines of five altitudes were carefully analyzed by comparing the reinforced and unreinforced slopes, covering the overall slope from top to bottom (Figure 10).

A close examination of displacement distribution at a horizontal line, $z=7.1 \mathrm{~cm}$, showed a significant difference between the reinforced and unreinforced slopes
(Figure 10(d)). For the pile-reinforced slope, an evident inflection occurred near the pile. On the left side of the inflection, the horizontal displacement increased significantly from the inner slope area to the piles, whereas this rate of increase became relatively small on the right side. On the other hand, for the unreinforced slope, the horizontal displacement increased from the inner slope at an approximately constant rate near the piles. It can be concluded that the piles significantly changed the displacement distribution of the slope at a certain area near the piles, and this inflection can be regarded as a boundary point to indicate that the piles significantly affected the deformation.

The inflections can be found in the displacement distribution curves of the reinforced slope at all altitudes (Figure 10), including the area above the piles (Figures 10(a)-10(c)). Therefore, a continuous surface was obtained by connecting these inflections using a curve, as shown in Figure 10 by the dotted line, denoted as the W-surface in this paper. The horizontal displacement of the pile-reinforced slope exhibited different distribution rules on different sides of the $W$-surface.

Similar to the horizontal displacement, the horizontal stress can also be used to reflect the effect of the piles on the slope. Figure 11 shows the horizontal stress distributions on horizontal lines at two typical altitudes, located near and above the piles, with the position of the $W$-surface being determined from the displacement distribution. It can be seen that the stress distribution curve exhibited a significant change near the $W$-surface. This demonstrated that the $W$ surface outlines the area where the piles have a significant effect on the slope, including the displacement and stress 

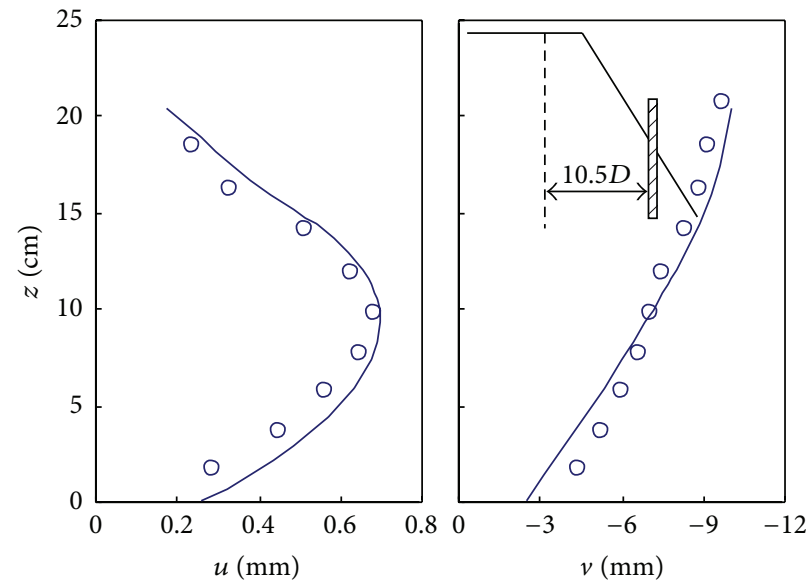

(a) $y=-10.5 D$

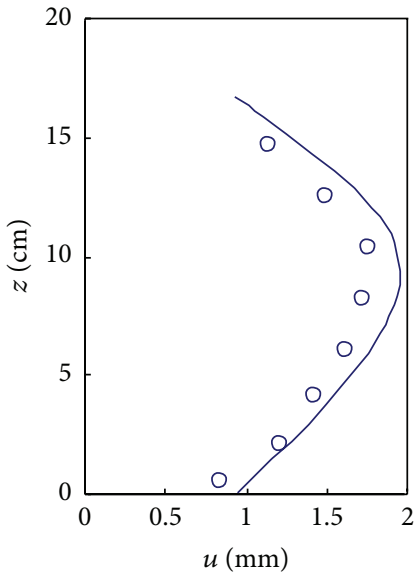

$u(\mathrm{~mm})$
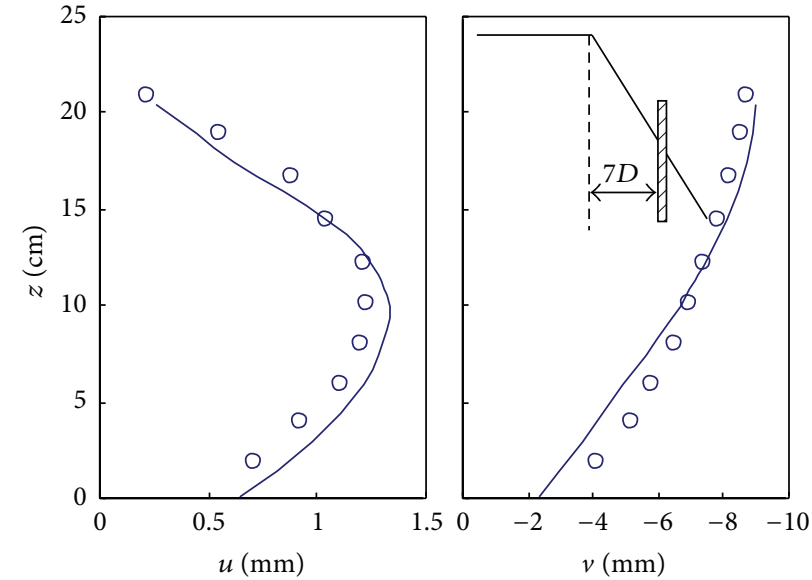

(b) $y=-7 D$, passing by slope shoulder

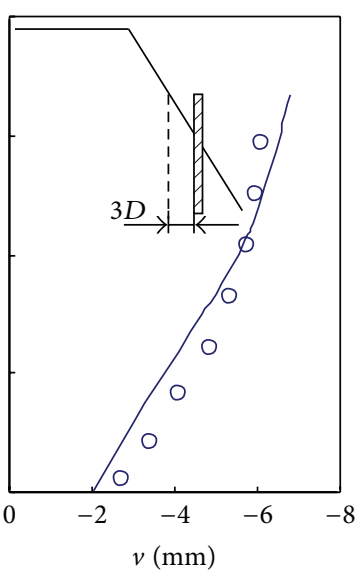

O Test

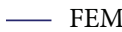

(c) $y=-3 D$

FIGURE 5: Displacement distribution comparison of numerical analysis and test results on different vertical sections. $u$ : horizontal displacement; $v$ : vertical displacement; $D$ : pile diameter.

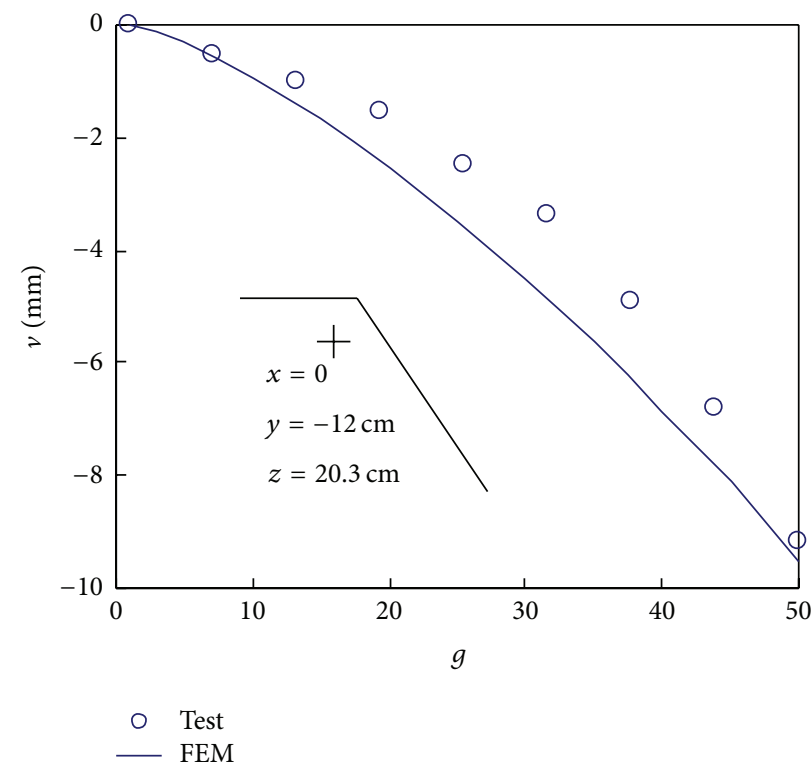

FIGURE 6: Vertical displacement history of a typical point by numerical analysis and test results. $v$ : vertical displacement; $g$ : g-level. 


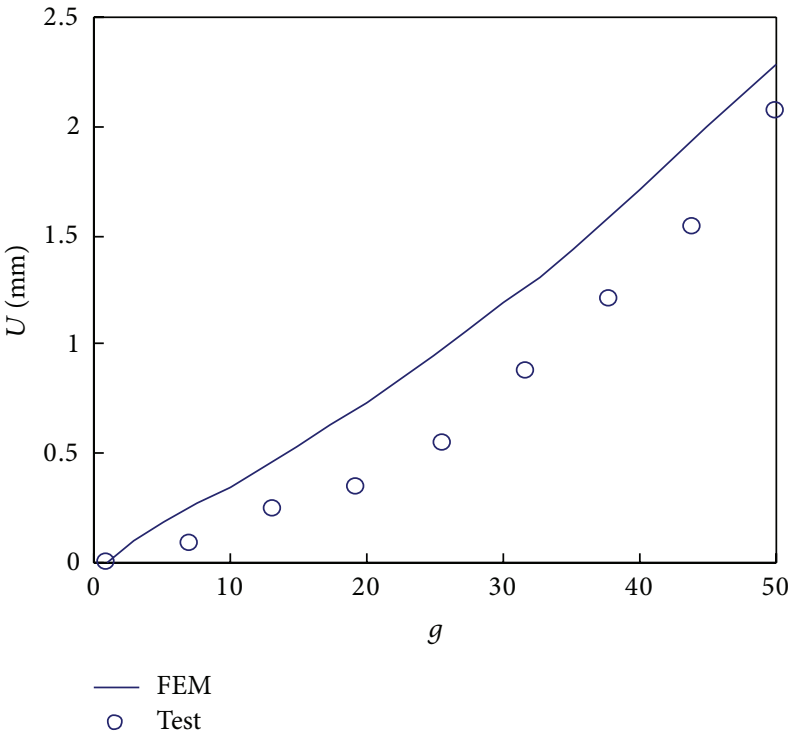

(a) Horizontal displacement of pile top

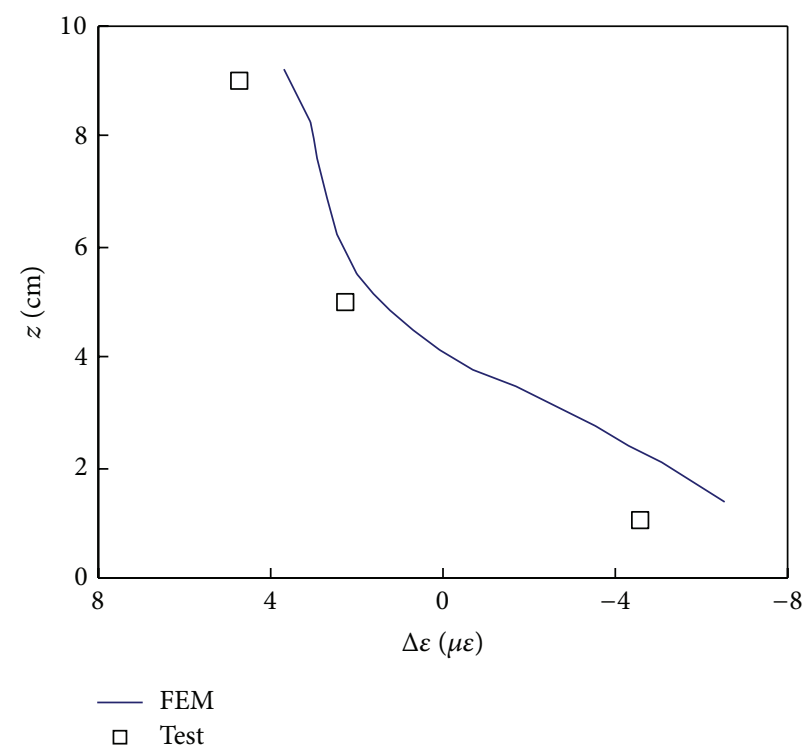

(b) Vertical strain distribution

FIGURE 7: Response of the pile by numerical analysis and test results. $u$ : horizontal displacement of pile top; $\Delta \varepsilon$ : vertical strain difference between right and left sides of pile.

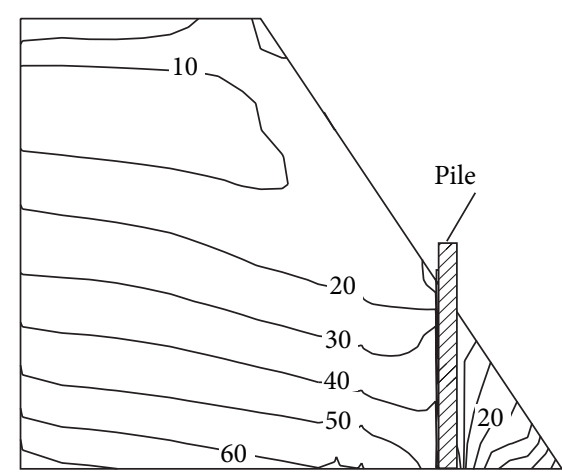

(a) Horizontal stress

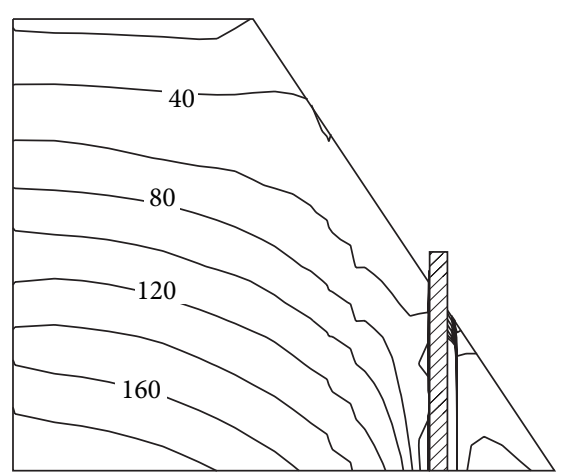

(b) Vertical stress

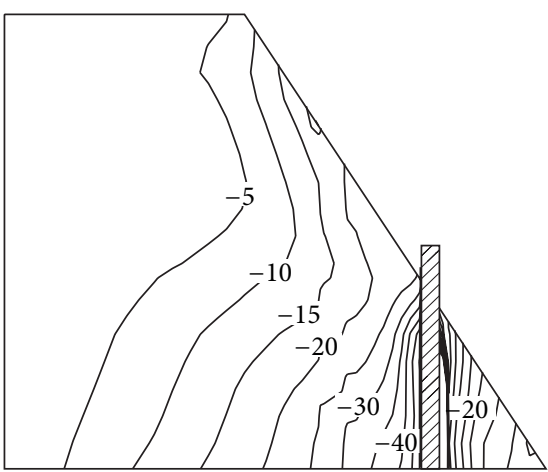

(c) Shear stress

FIGURE 8: Stress distribution of the slope at $50 \mathrm{~g}$-level by numerical analysis (unit: $\mathrm{kPa}$ ).

response. Thus, the $W$-surface can be used as an important index to describe the influence of piles on stress-deformation behavior of the reinforced slope.

4.2. Division of Zones. According to the $W$-surface, the reinforced slope behind the piles can be divided into two zones: one is where the slope is significantly affected by the piles, while the other is where the slope is insignificantly affected. The former was further examined by using the horizontal stress distribution of the slope at different vertical sections (Figure 12). The horizontal stress decreased with increasing altitude from the slope bottom; however, there was a significant inflection in the upper part of the slope. From this inflection, the change trend of the stress noticeably altered. It can be concluded that the stress states of the slope were significantly different on the different sides of the inflection, and the reason can be attributed to the effect of the piles.

Thus, another continuous surface was yielded by connecting these inflections using a curve, as the dotted line showed in Figure 12. This surface, denoted as the L-surface in this paper, indicates the boundary where the effect of the piles exhibited different features on different sides. Accordingly, the slope can be divided into three zones behind the piles according to these two surfaces (Figure 13).

The stress-deformation behavior exhibited different features in different zones. Zone A is the area at the left side of the $W$-surface, far from the piles. Thus, the piles had a small effect on this zone. For example, the horizontal displacement of the reinforced slope showed a similar distribution to the unreinforced slope in zone A. Zone B is above the L-surface and has a free surface. The change of deformation of the slope 


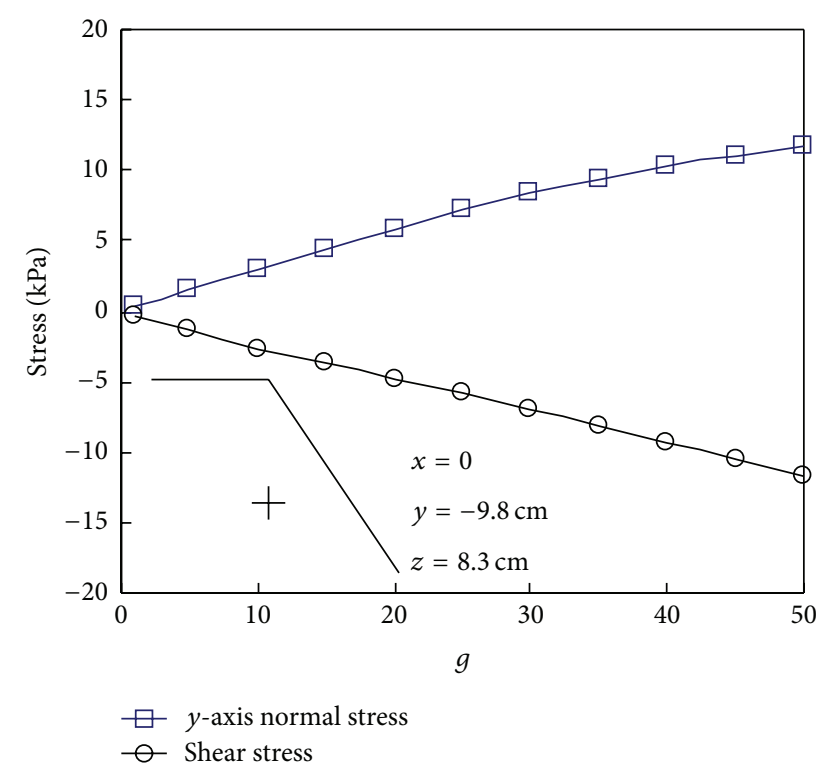

FIGURE 9: Stress history of a typical point by numerical analysis. $g$ : g-level.

became flat in this zone; this demonstrated that the piles significantly restricted the deformation. Zone $\mathrm{C}$ is at the right side of the $W$-surface and directly contacts the piles. The piles significantly reduced the horizontal displacement of the soil in this zone; thus, the displacement distribution showed a flat curve. Moreover, the horizontal stress exhibited a significant change with increasing distance from the piles. This demonstrated that the piles induced significant compression effect in this zone.

\section{Reinforcement Mechanism}

The strain of a soil element within the slope was introduced to analyze the pile-reinforcement mechanism of the slope by comparing the reinforced and unreinforced slopes. This strain can be determined using the measured displacement of the centrifuge model tests because the measurement may be more reliable than the numerical result, especially at the failure state of the unreinforced slope. A two-dimensional, four-node square isoparametric element, $1 \mathrm{~cm}$ long, was used for strain analysis. The strain at the center was thought to be the one of this element that was assumed to be uniform within the element.

The deformation of soil can be divided into two independent components owing to shear and compression applications. This implies that shear and compression play different roles in the deformation and failure of a slope. For example, the formation of a slip surface may significantly depend on the increase in shear strain, whereas a tensional crack, which commonly occurs at the top of a slope, is induced mainly by a decrease in compression strain. It can be concluded that the piles significantly changed the strain state of the slope and thus increased the stability level of the slope. Therefore, the pile-reinforcement mechanism can be analyzed using the effects of the piles on the shear and compression strains.

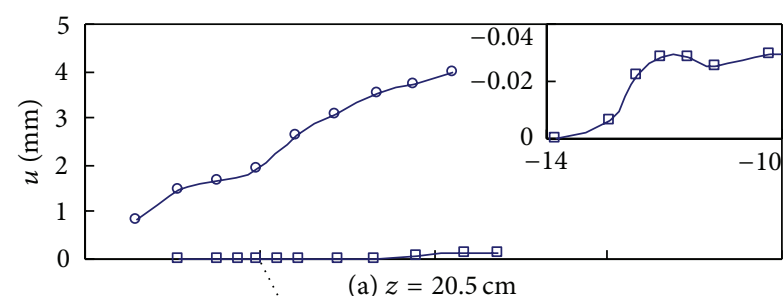

(a) $z=20.5 \mathrm{~cm}$

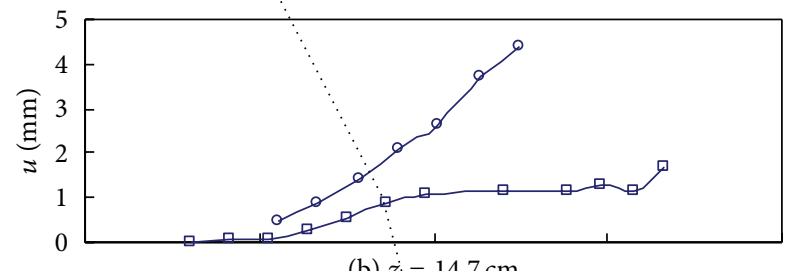

(b) $\dot{z}=14.7 \mathrm{~cm}$
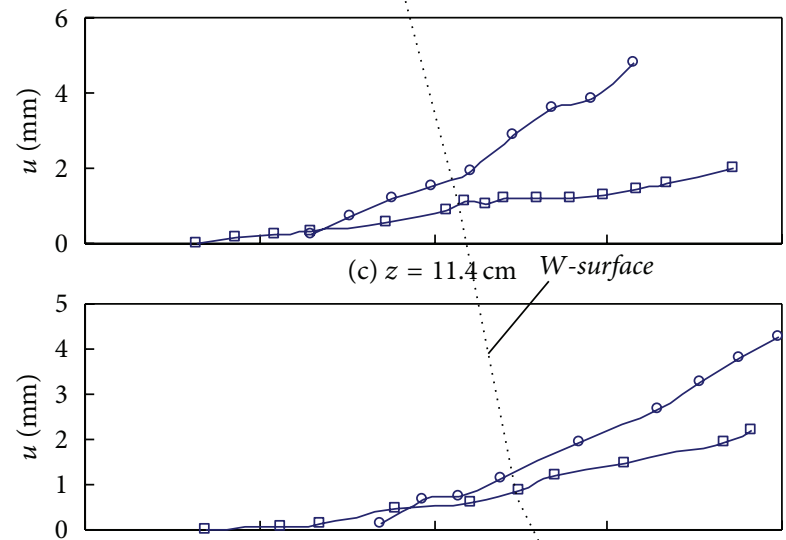

(d) $z=7.1 \mathrm{~cm}$

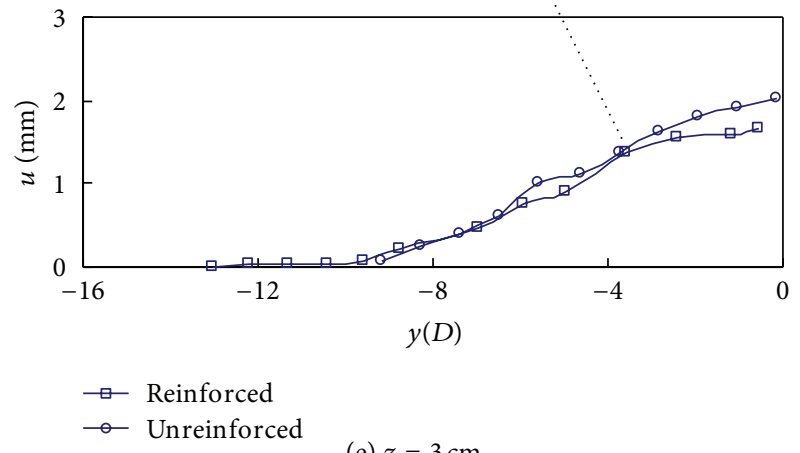

(e) $z=3 \mathrm{~cm}$

FIGURE 10: Horizontal displacement distributions on horizontal sections at $50 \mathrm{~g}$-level by test observation. $u$ : horizontal displacement; $D$ : pile diameter.

The deformation due to shear was indicated by the slopedirection shear strain. The horizontal compression strain was used to indicate the deformation due to compression. Figure 14 shows the strain histories of typical elements in the three zones (positions are shown in Figure 13); the elements of the unreinforced slope, corresponding to those in the reinforced slope, are also presented for comparison. It should be noted that the horizontal compression strains of these elements were all negative, indicating dilation in the horizontal direction.

In zone B, the shear strain of a typical element in the unreinforced slope increased rapidly with increasing centrifugal 


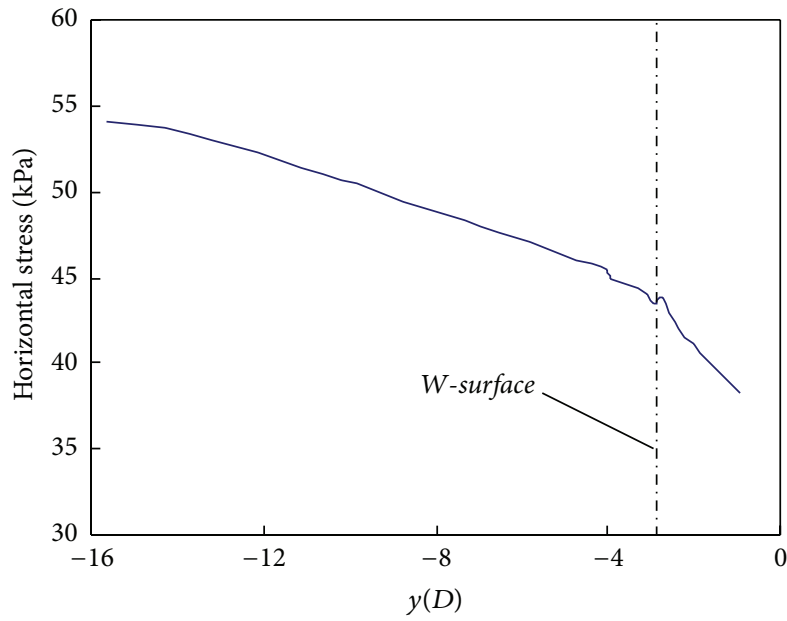

(a) $z=3.5 \mathrm{~cm}$

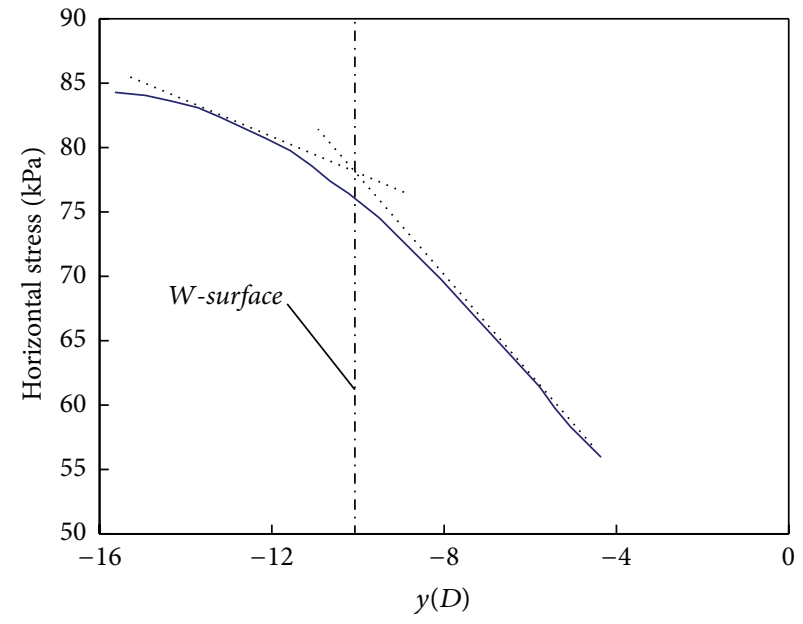

(b) $z=16.5 \mathrm{~cm}$

FIGURE 11: Stress distribution on a horizontal line by numerical analysis.

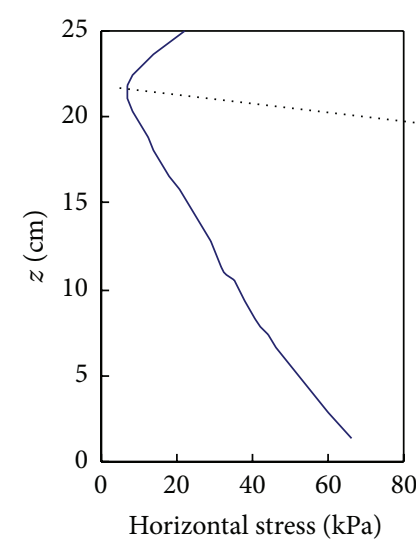

(a) $y=-10.5 \mathrm{D}$

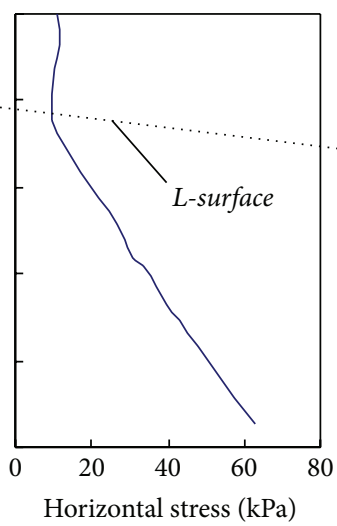

(b) $y=-7.5 D$

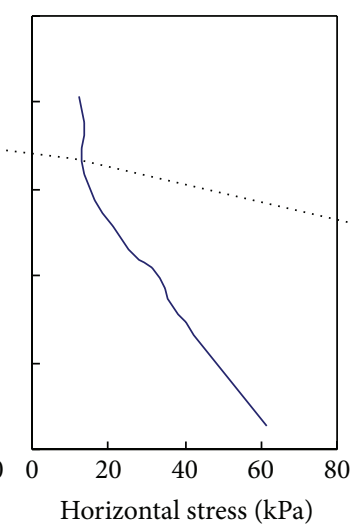

(c) $y=-4.5 \mathrm{D}$

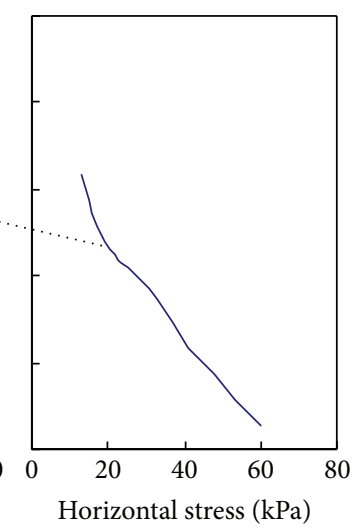

(d) $y=-2.5 \mathrm{D}$

FIGURE 12: Stress distributions on different vertical sections at 50 g-level by numerical analysis.

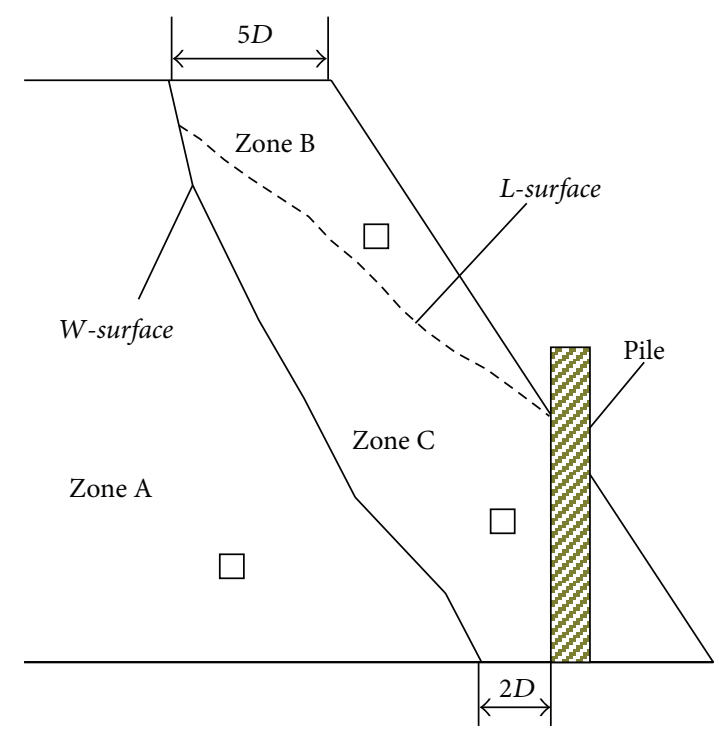

FIgURE 13: Division of zones with different features. $D$ : pile diameter. 

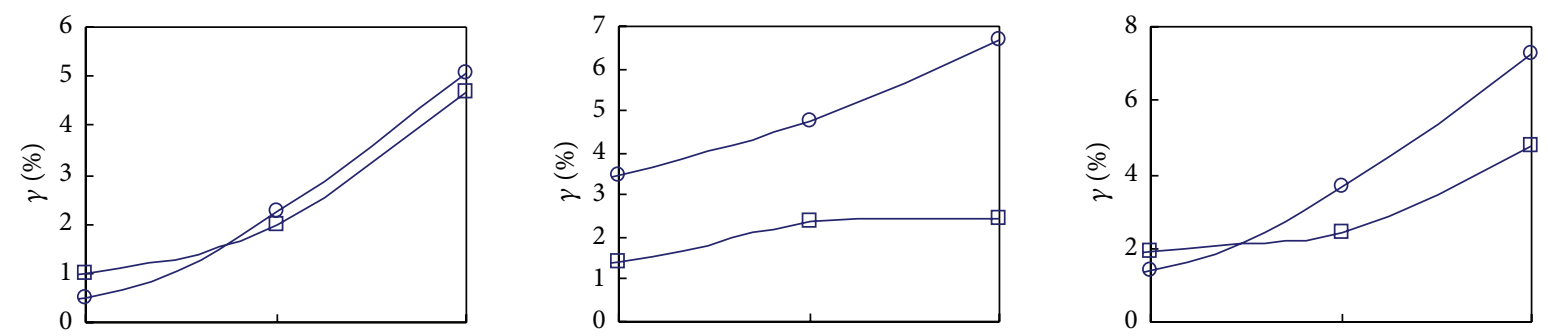

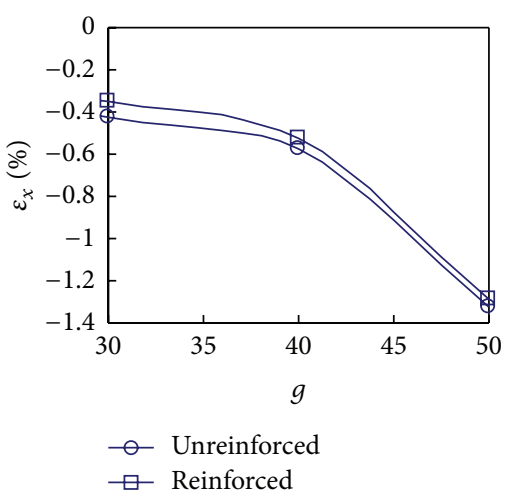

(a) Zone A

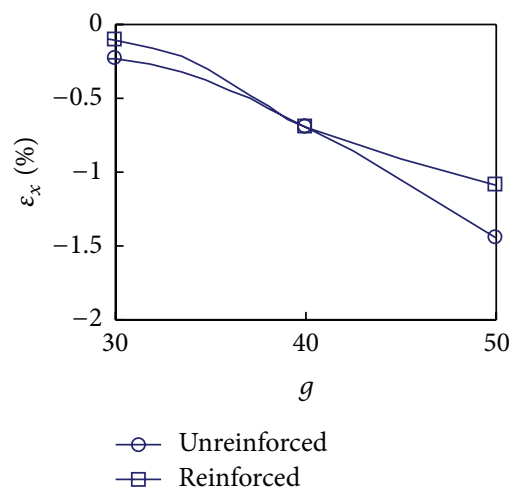

(b) Zone B

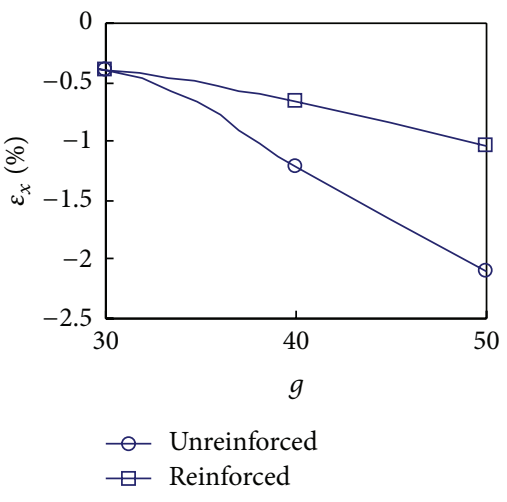

(c) Zone C

FIGURE 14: Strain history of typical elements of different zones by test observation. $\gamma$ : slope-direction shear strain; $\varepsilon_{x}$ : horizontal compression strain; $g$ : g-level.

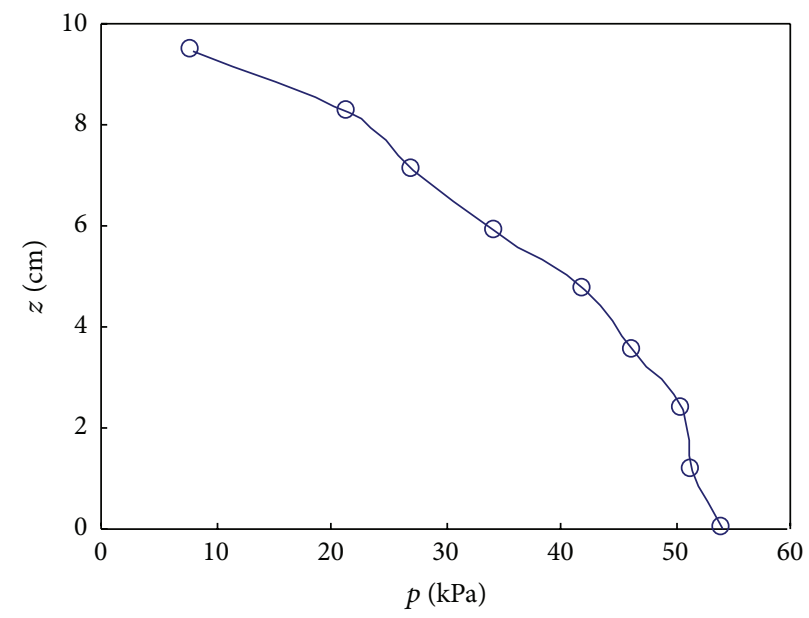

FIGURE 15: Earth pressure on the pile by numerical analysis. $p$ : earth pressure.

acceleration (Figure 14(b)). This strain reached a significant magnitude at $50 \mathrm{~g}$-level when the landslide, just across this element, occurred. However, the shear strain increased at a smaller rate and reached a lower level far from failure if the piles were used. Thus, a basic concept, shear effect, was introduced to describe that shear strain was decreased due to the effect of the piles. In other words, a significant shear effect of piles in zone $B$ arrested the formation of a slip surface. Accordingly, the horizontal compression strain increased due to the piles, which was described using another basic concept, compression effect. There was also significant compression effect of piles in zone B; however, it can be concluded that the shear effect was primary.

The history of the compression strain showed that this strain in the reinforced slope was significantly larger than that of the unreinforced one; this demonstrated that there was significant compression effect of piles in zone C (Figure 14(c)). Closer examination showed that the compression effect was more significant than the shear effect, though the shear effect of the piles was also distinct.

The compression and shear strains both exhibited minor differences in zone $\mathrm{A}$ for the reinforced and unreinforced 


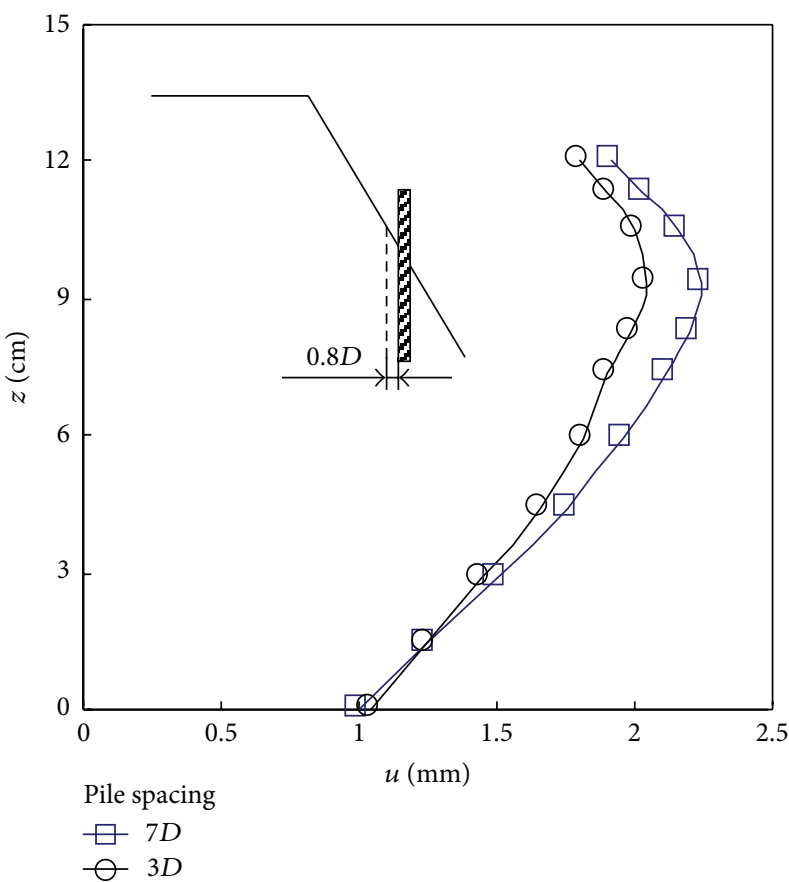

(a) $y=-0.8 D$

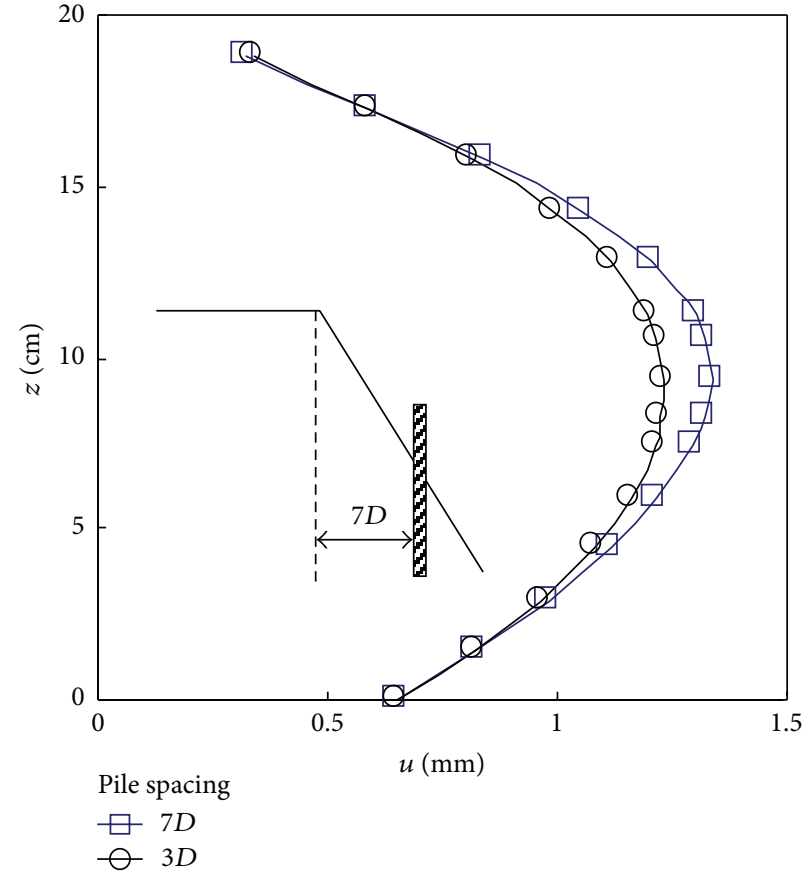

(b) $y=-7 D$, passing by slope shoulder

FIGURE 16: Influence of pile spacing by numerical analysis. $D$ : pile diameter; $u$ : horizontal displacement.

slopes (Figure 14(a)); this indicated that both the shear effect and compression effect were negligible in this zone.

Figure 15 shows the earth pressure on the inner side of the pile, obtained by numerical analysis. The earth pressure decreased with increasing altitude, similar to a distribution of earth pressure for a retaining wall. Combined with the strain analysis results at the overall slope level, the reinforcement mechanism can be demonstrated using the shear effect and compression effect of piles, which were of different levels in different zones, as follows: the pile and neighboring soil exhibited a significant interaction due to loading. This interaction was transferred to the adjacent zone and induced a significant compression effect (zone C). This compression effect transferred upwards and caused a prominent shear effect (zone B), arresting the possible sliding and increasing the stability level of the slope.

\section{Influence Factors}

The numerical analysis was used to compute different cases to discuss the influence factors of the behavior of pile-reinforced slopes by altering several factors based on the centrifuge test condition, including pile spacing, pile location, restriction style of pile end, and inclination of slope. According to previous analysis, the horizontal displacement of the lateral side of the slope that the piles pass across is used as the characteristic index to illustrate the effect of these factors.

6.1. Pile Spacing. The pile spacing was reduced to $3 D$ (pile diameter) from approximately $7 D$ of the test condition. Figure 16 compares the horizontal displacements according to the different pile spacings, both obtained from numerical analysis, to discuss the influence rules. The horizontal displacement of the slope exhibited a small decrease when the pile spacing was reduced; such a difference decreased with increasing distance from the piles. The fundamental rules of horizontal displacement were consistent for different pile spacings.

6.2. Location of Pile. The piles were moved upwards several centimeters in the comparison case, as described using the dashed line in Figure 1(b). The horizontal displacements according to new and original locations of piles were obtained from numerical analysis (Figure 17). The horizontal displacement of the slope exhibited a significant decrease near the piles if the pile was located at an upper position, which may be partly attributed to the new location of piles being farther from the free surface of the slope. The difference decreased with increasing distance from the slope surface and can be ignored at the section passing by the slope shoulder (Figure 17(c)). It can be derived that the distributions of horizontal displacement of the pile-reinforced slope were consistent for different pile locations.

6.3. Restriction Style of Pile End. The pile ends were all fixed on the container to prevent the relative movement between the piles and the container bottom, different from the test condition that the piles may move along the container bottom. Figure 18 compares the horizontal displacements according to the different restriction styles, both obtained from numerical analysis. The horizontal displacement of 


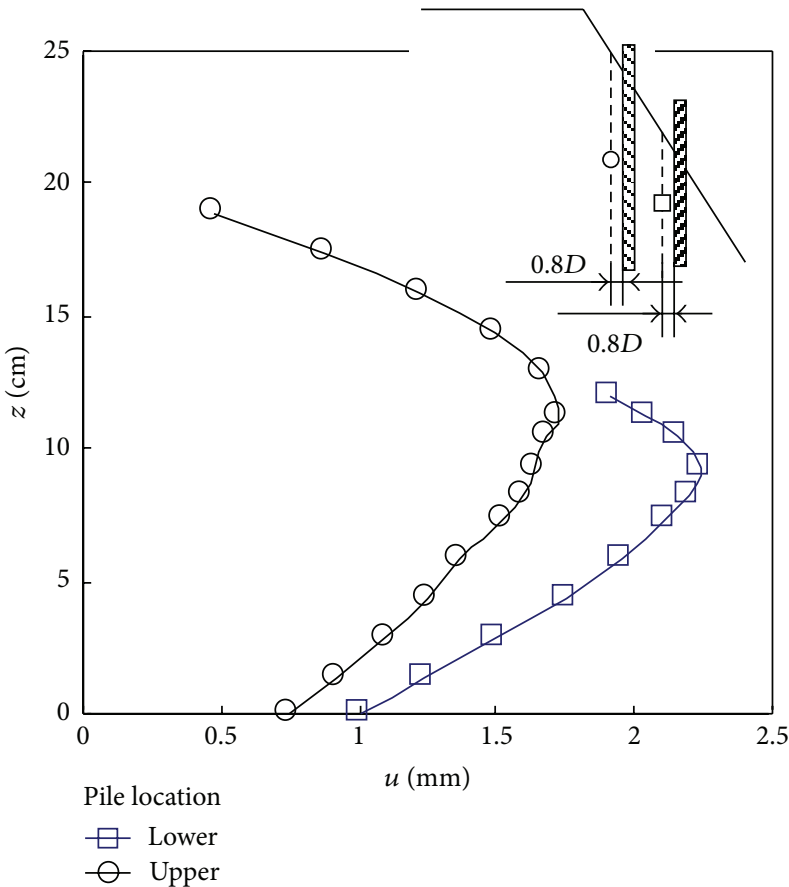

(a) $0.8 D$ from pile

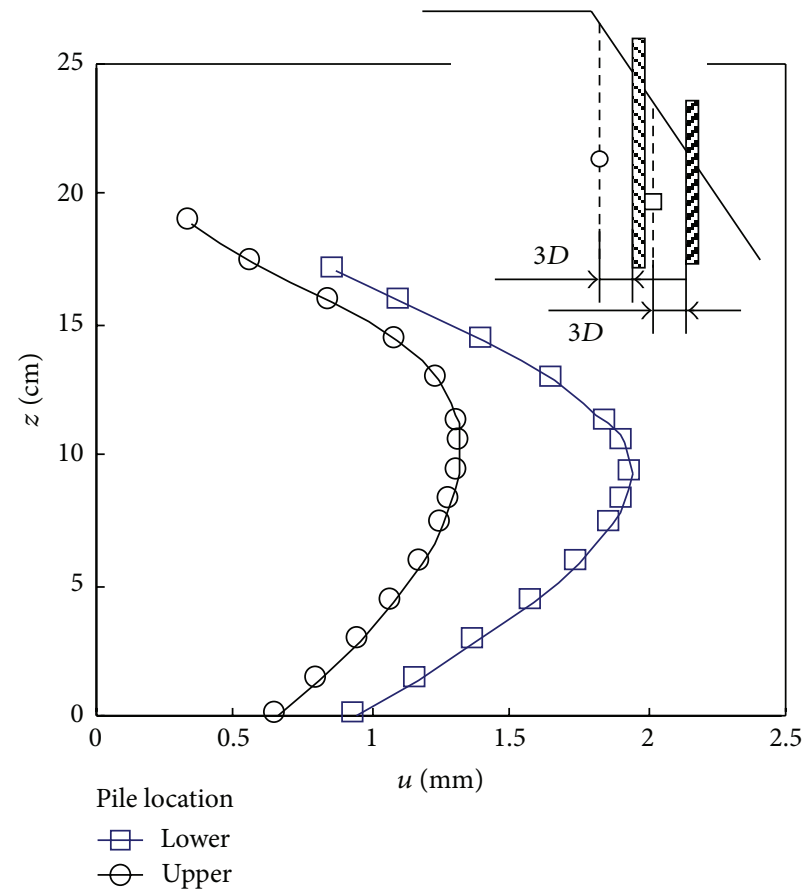

(b) $3 D$ from pile

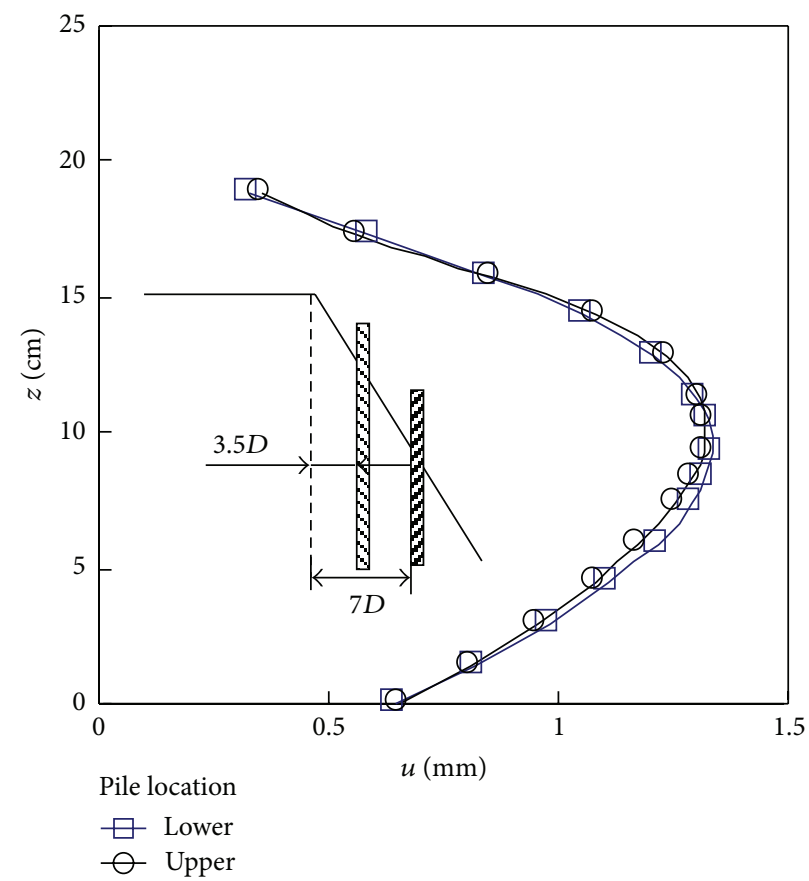

(c) $y=-7 D$, passing by slope shoulder

FIGURE 17: Influence of positions of the pile by numerical analysis. $D$ : pile diameter; $u$ : horizontal displacement.

the slope significantly decreased, especially near the piles, when the pile ends were fixed. Overall, the fundamental rules of horizontal displacement of pile-reinforced slope can be found to be consistent regardless whether the restriction style of pile end was altered.

6.4. Inclination of Slope. The inclination of the slope was changed to $1: 1$ from the original $1.5: 1$ in the test condition, and the relative location of piles was maintained as in the original scheme. The horizontal displacements according to the different inclinations of slopes were yielded from numerical analysis (Figure 19). The horizontal displacement of the slope exhibited an evident decrease due to the decrease of inclination of slope. However, the distribution rules of horizontal displacement were consistent for the different inclinations of slope. 


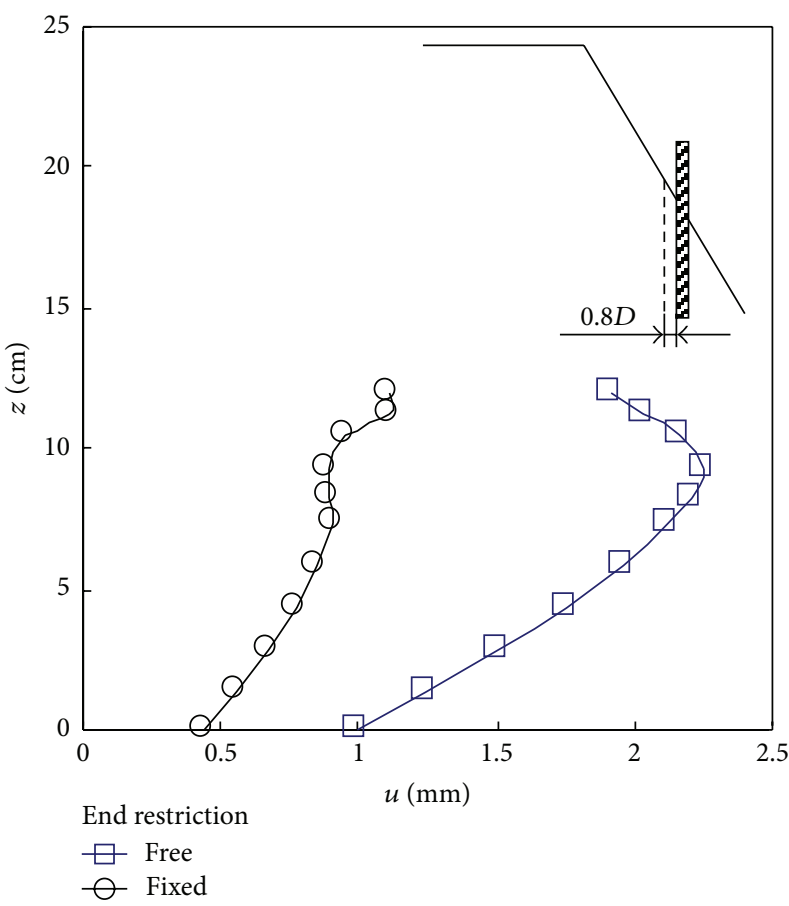

(a) $y=-0.8 D$

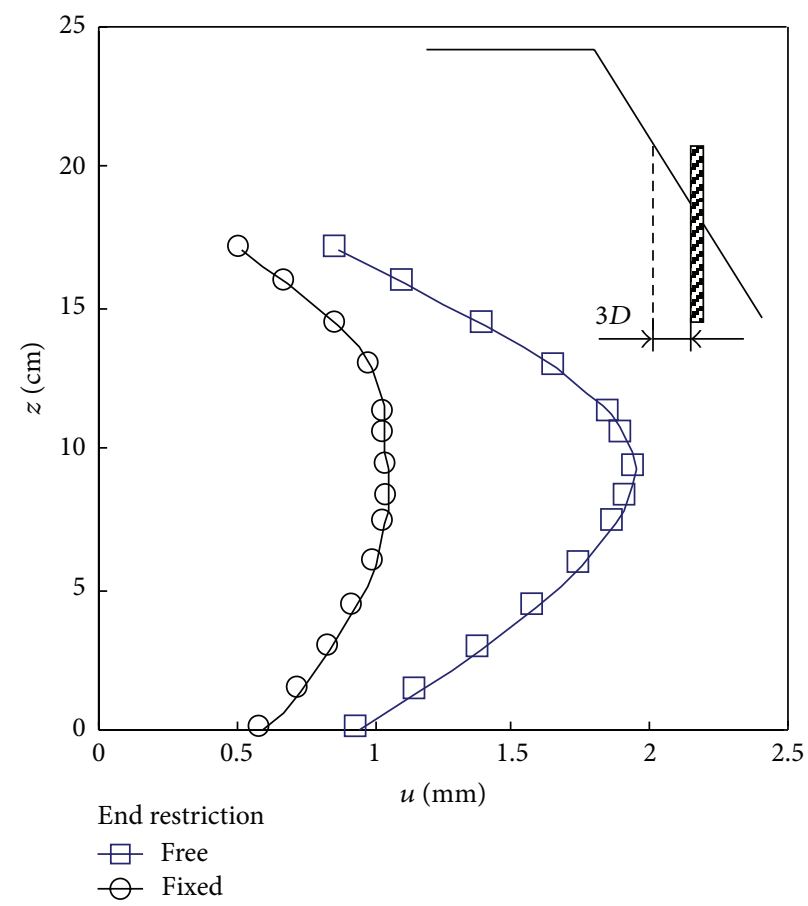

(b) $y=-3 D$

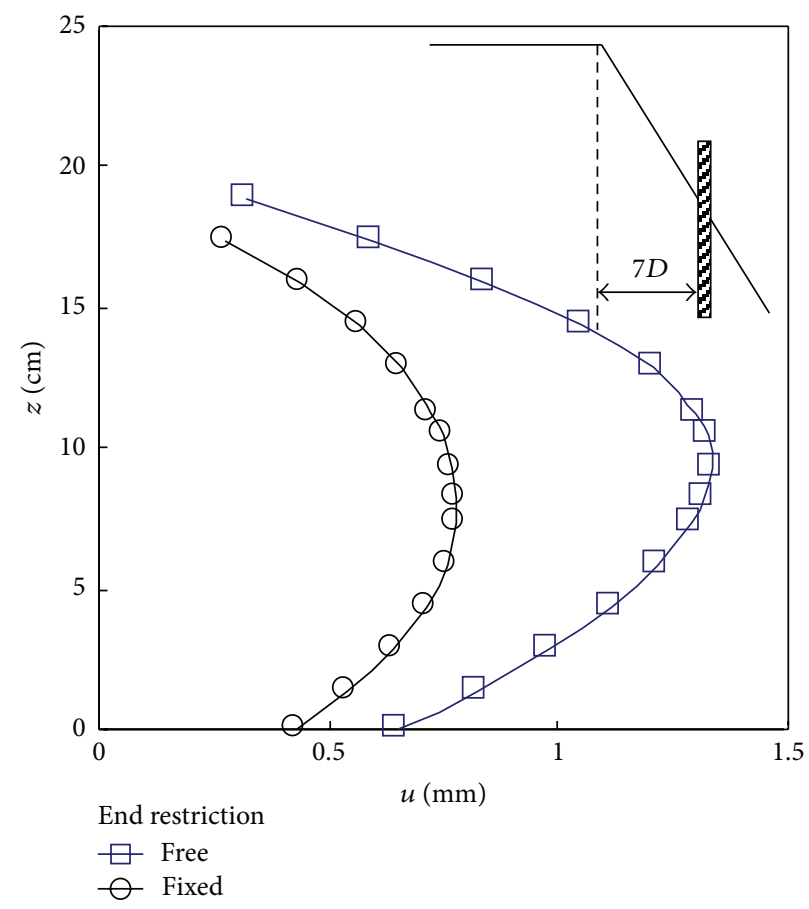

(c) $y=-7 D$, passing by slope shoulder

FIGURE 18: Influence of fixing styles of the pile end by numerical analysis. $D$ : pile diameter; $u$ : horizontal displacement.

6.5. Summary. Based on the numerical analysis, it can be concluded that the behavior of the pile-reinforced slopes was similar for the different factors considered in this paper. As discussed in above texts, the two boundary surfaces, $W$ surface and L-surface, can be used as important indicators of the behavior of reinforced slope. Therefore, these surfaces were summarized corresponding to different factors according to the results from numerical analysis (Figure 20). It can be seen that both of the surfaces can be described using a definite format, though their positions and curvatures were affected by different factors. Thus, it is believed that the fundamental rules and reinforcement mechanism, derived in 


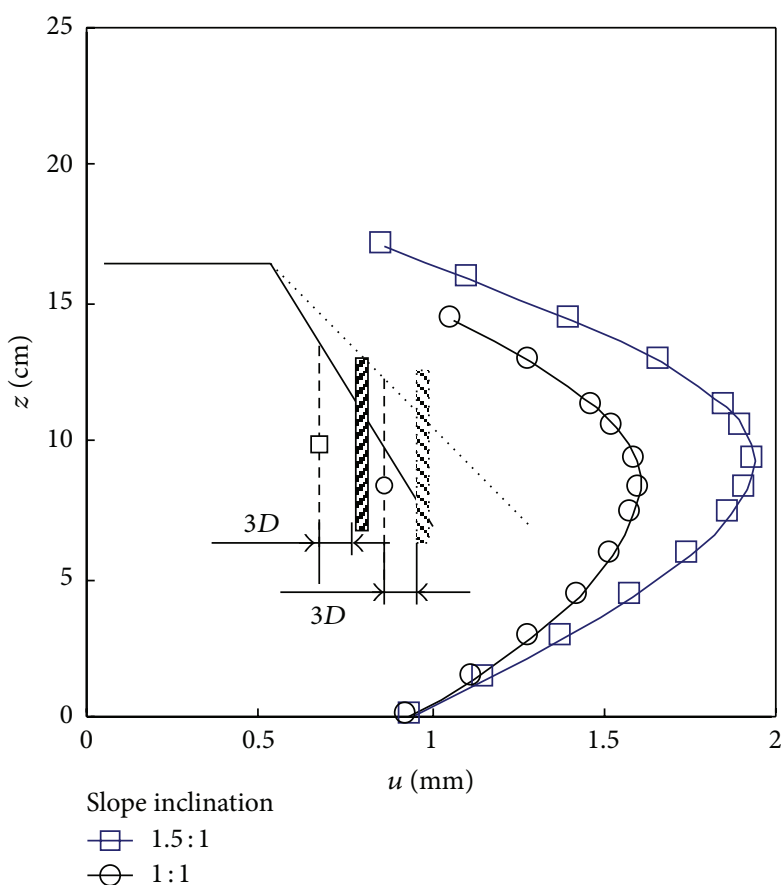

(a) $3 D$ from pile

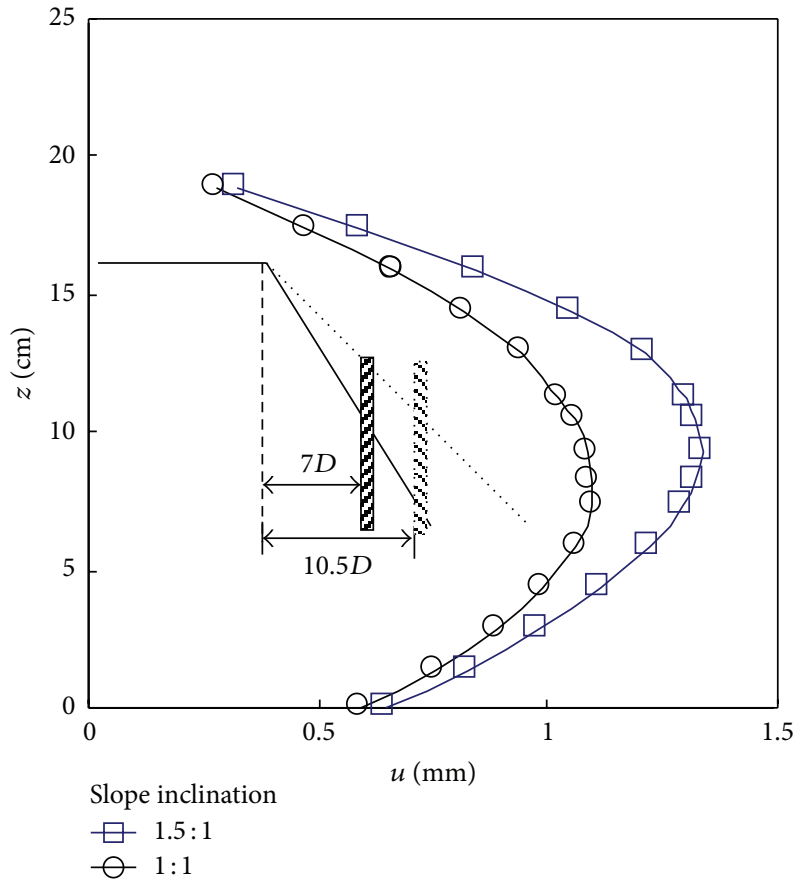

(b) $y=-7 D$, passing by slope shoulder

FIGURE 19: Influence of inclination of the slope by numerical analysis. $D$ : pile diameter; $u$ : horizontal displacement.

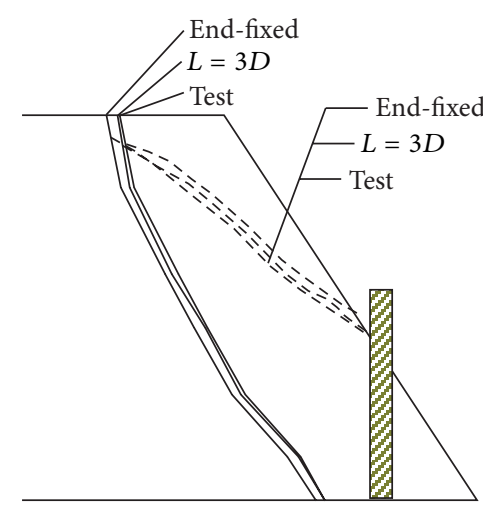

- W-surface
-- L-surface

(a) Test, $L=3 D$, pile end fixed
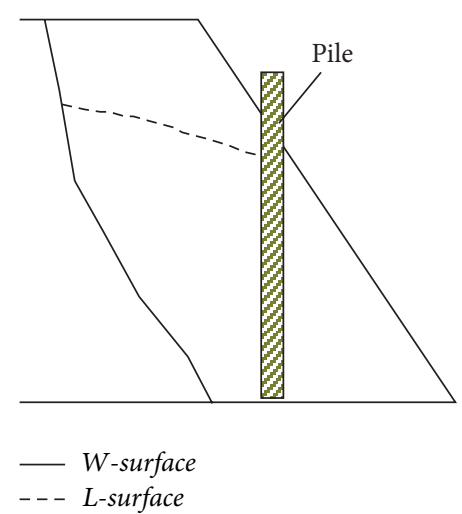

(b) Pile location changed

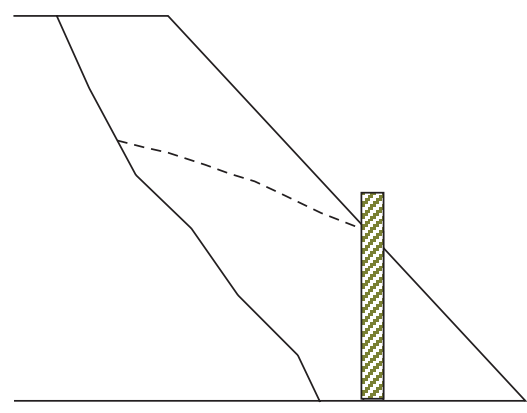

- W-surface
-- - L-surface

(c) Slope inclination changed to $1: 1$

Figure 20: $W$-surfaces and $L$-surfaces considering different factors. $L$ : pile spacing; $D$ : pile diameter.

this paper, may be suitable for most practical pile-reinforced slopes.

\section{Conclusions}

The pile-reinforcement behavior of cohesive soil slope was investigated using numerical analysis and centrifuge model tests. The behavior and reinforcement mechanism of the pile-reinforced slope were obtained by considering different influence factors. The main conclusions are as follows.
(1) The piles arrested a landslide that occurred in an unreinforced slope, thus, significantly increasing the stability level of the slope.

(2) A numerical model was established and confirmed to be effective in capturing primary behavior of the pile-reinforced slope. Thus, a comprehensive understanding of stress-deformation response can be achieved by combining the numerical and physical simulations. 
(3) The piles have a significant effect on the behavior of reinforced slope; the boundary of the influenced area can be described using a continuous surface.

(4) The pile-reinforcement mechanism can be described using two basic concepts: compression effect and shear effect, which, respectively, refer to the piles increasing the compression strain and decreasing the shear strain in comparison with unreinforced slope. The pile-soil interaction induces significant compression effect in the inner zone near the piles; this effect is transferred to the upper part of the slope, with the shear effect becoming prominent. As a result, possible sliding is prevented; the stability level of the slope is accordingly increased.

(5) The response of pile-reinforced slope was significantly affected by several factors such as pile spacing, pile location, restriction style of pile end, and inclination of slope; however, the behavior and reinforcement mechanism are consistent for the different factors considered in this paper.

\section{Acknowledgments}

The study is supported by National Natural Science Foundation of China (nos. 51129902, 50979045) and the State Key Laboratory of Hydroscience and Engineering (no. 2013-KY$5)$.

\section{References}

[1] C. D. F. Rogers and S. Glendinning, "Improvement of clay soils in situ using lime piles in the UK," Engineering Geology, vol. 47, no. 3, pp. 243-257, 1997.

[2] J. A. Smethurst and W. Powrie, "Monitoring and analysis of the bending behaviour of discrete piles used to stabilise a railway embankment," Geotechnique, vol. 57, no. 8, pp. 663-677, 2007.

[3] M. Kimura, S. Inazumi, J. K. A. Too, K. Isobe, Y. Mitsuda, and Y. Nishiyama, "Development and application of $\mathrm{H}$-joint steel pipe sheet piles in construction of foundations for structures," Soils and Foundations, vol. 47, no. 2, pp. 237-251, 2007.

[4] T. Ito and T. Matsui, "Methods to estimate lateral force acting on stabilizing piles," Soils and Foundations, vol. 15, no. 4, pp. 43-59, 1975.

[5] H. G. Poulos, "Design of reinforcing piles to increase slope stability," Canadian Geotechnical Journal, vol. 32, no. 5, pp. 808818, 1995.

[6] M. A. El Sawwaf, "Strip footing behavior on pile and sheet pilestabilized sand slope," Journal of Geotechnical and Geoenvironmental Engineering, vol. 131, no. 6, pp. 705-715, 2005.

[7] M. J. Thompson and D. J. White, "Design of slope reinforcement with small-diameter piles," in Advances in Earth Structures: Research to Practice, GSP 151, pp. 67-73, ASCE, 2006.

[8] A. Bouafia and A. Bouguerra, "Centrifuge testing of the behaviour of a horizontally loaded flexible pile near to a slope," Canadian Geotechnical Journal, vol. 32, no. 2, pp. 324-335, 1995.

[9] A. Porbaha and D. J. Goodings, "Centrifuge modeling of geotextile-reinforced steep clay slopes," Canadian Geotechnical Journal, vol. 33, no. 5, pp. 696-704, 1996.
[10] J. G. Zornberg, N. Sitar, and J. K. Mitchell, "Performance of geosynthetic reinforced slopes at failure," Journal of Geotechnical and Geoenvironmental Engineering, vol. 124, no. 8, pp. 670683, 1998.

[11] N. I. Thusyanthan, S. P. G. Madabhushi, and S. Singh, "Tension in geomembranes on landfill slopes under static and earthquake loading-Centrifuge study," Geotextiles and Geomembranes, vol. 25, no. 2, pp. 78-95, 2007.

[12] L. Wang, G. Zhang, and J.-M. Zhang, "Nail reinforcement mechanism of cohesive soil slopes under earthquake conditions," Soils and Foundations, vol. 50, no. 4, pp. 459-469, 2010.

[13] L. P. Wang and G. Zhang, "Centrifuge model test study on pile reinforcement behavior of cohesive soil slopes under earthquake conditions," Landslides, 2013.

[14] T. Yamagami, J. Jiang -C, and K. Ueno, "A limit equilibrium stability analysis of slopes with stabilizing piles," in Advances in Earth Structures: Research to Practice, GSP 101, pp. 343-354, ASCE, 2000.

[15] G. Zhang and L. Wang, "Stability analysis of strain-softening slope reinforced with stabilizing piles," Journal of Geotechnical and Geoenvironmental Engineering, vol. 136, no. 11, pp. 15781582, 2010.

[16] E. Ausilio, E. Conte, and G. Dente, "Stability analysis of slopes reinforced with piles," Computers and Geotechnics, vol. 28, no. 8, pp. 591-611, 2001.

[17] F. Cai and K. Ugai, "Numerical analysis of the stability of a slope reinforced with piles," Soils and Foundations, vol. 40, no. 1, pp. 73-84, 2000.

[18] S. Jeong, B. Kim, J. Won, and J. Lee, "Uncoupled analysis of stabilizing piles in weathered slopes," Computers and Geotechnics, vol. 30, no. 8, pp. 671-682, 2003.

[19] J. Won, K. You, S. Jeong, and S. Kim, "Coupled effects in stability analysis of pile-slope systems," Computers and Geotechnics, vol. 32, no. 4, pp. 304-315, 2005.

[20] C. W. W. Ng and L. M. Zhang, "Three-dimensional analysis of performance of laterally loaded sleeved piles in sloping ground," Journal of Geotechnical and Geoenvironmental Engineering, vol. 127, no. 6, pp. 499-509, 2001.

[21] C. W. W. Ng, L. M. Zhang, and K. K. S. Ho, "Influence of laterally loaded sleeved piles and pile groups on slope stability," Canadian Geotechnical Journal, vol. 38, no. 3, pp. 553-566, 2001.

[22] G. R. Martin and C.-Y. Chen, "Response of piles due to lateral slope movement," Computers and Structures, vol. 83, no. 8-9, pp. 588-598, 2005.

[23] G. Zhang, Y. Hu, and J.-M. Zhang, "New image analysis-based displacement-measurement system for geotechnical centrifuge modeling tests," Measurement, vol. 42, no. 1, pp. 87-96, 2009.

[24] G. Zhang, D. Liang, and J.-M. Zhang, "Image analysis measurement of soil particle movement during a soil-structure interface test," Computers and Geotechnics, vol. 33, no. 4-5, pp. 248-259, 2006.

[25] G. Zhang, J.-M. Zhang, and Y. Yu, "Modeling of gravelly soil with multiple lithologic components and its application," Soils and Foundations, vol. 47, no. 4, pp. 799-810, 2007.

[26] G. Zhang and J.-M. Zhang, "Unified modeling of monotonic and cyclic behavior of interface between structure and gravelly soil," Soils and Foundations, vol. 48, no. 2, pp. 231-245, 2008.

[27] G. Zhang and J.-M. Zhang, "Numerical modeling of soilstructure interface of a concrete-faced rockfill dam," Computers and Geotechnics, vol. 36, no. 5, pp. 762-772, 2009. 


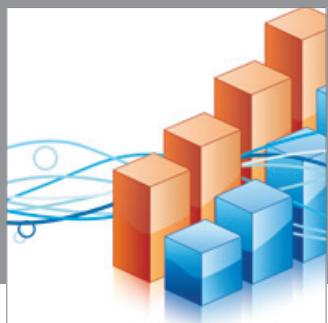

Advances in

Operations Research

mansans

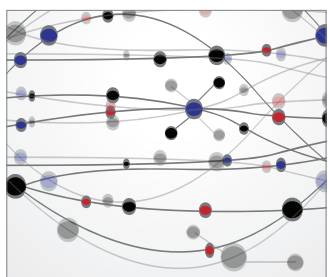

The Scientific World Journal
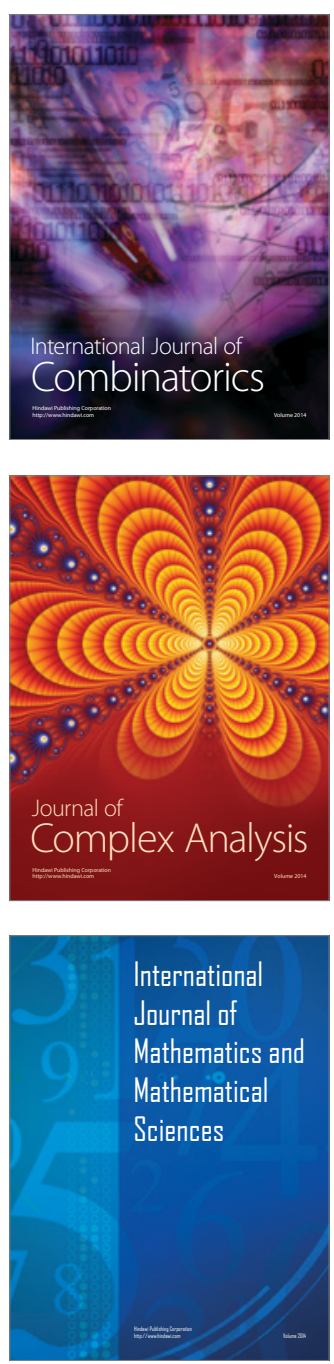
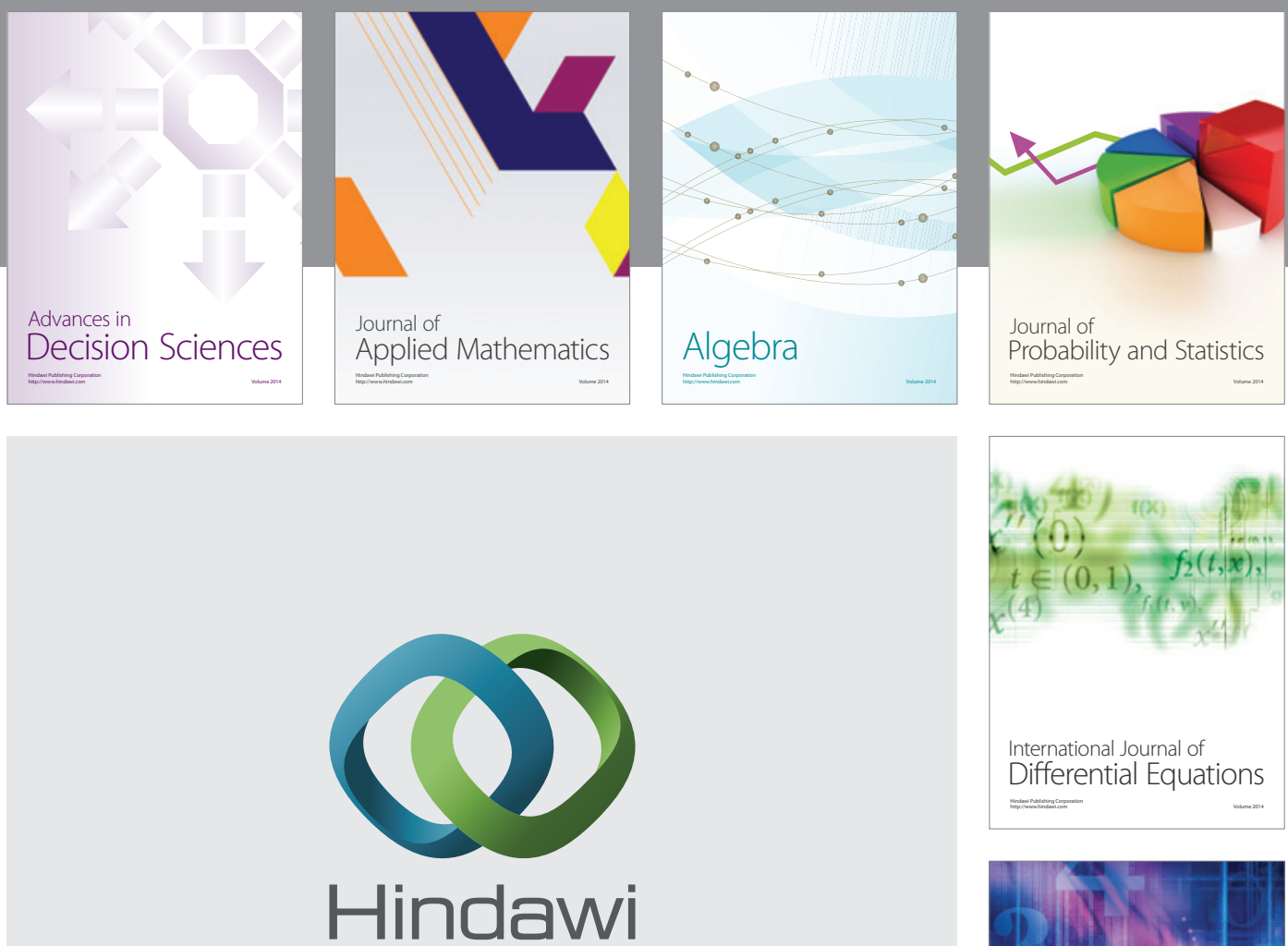

Submit your manuscripts at http://www.hindawi.com
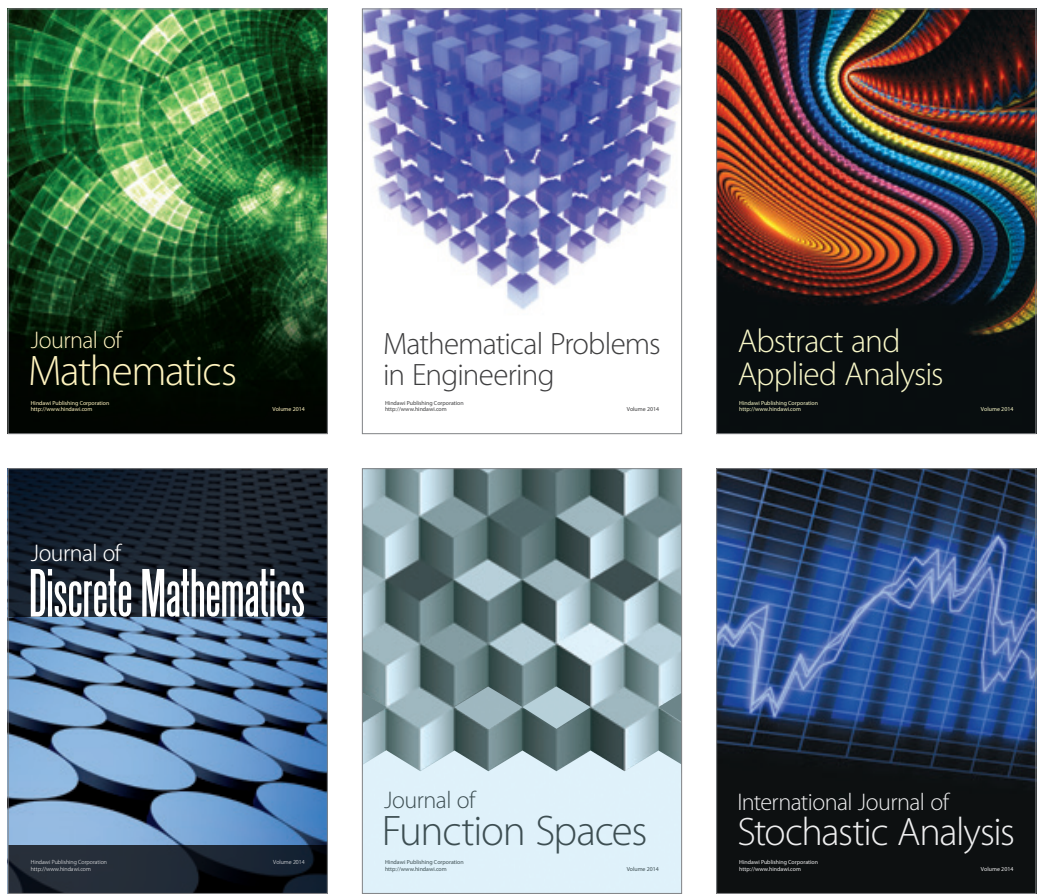

Journal of

Function Spaces

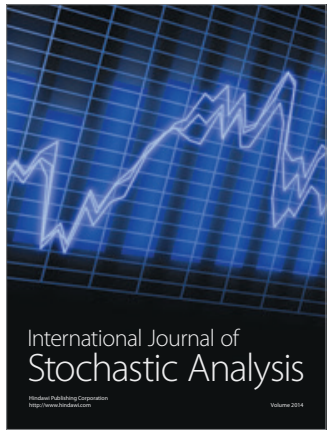

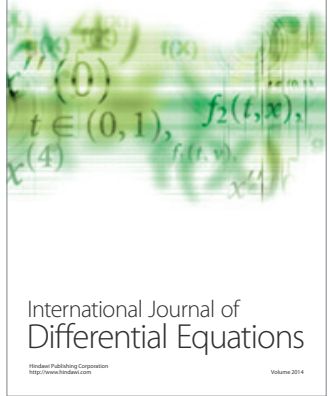
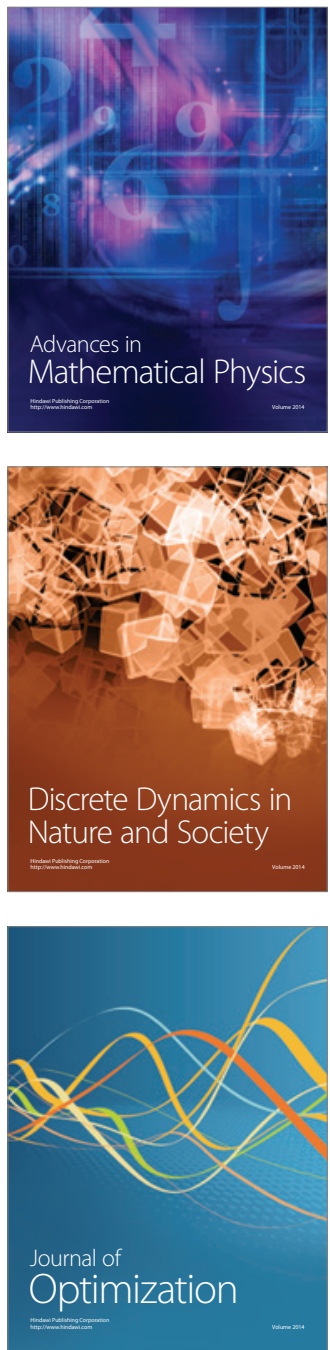